\title{
The Interpretive Shaping of Embodied Musical Structure in Piano Performance
}

\author{
BRYONY BUCK [1] \\ School of Science \& Engineering, University of Glasgow, UK \\ JENNIFER MACRITCHIE [1] \\ Conservatorio della Svizzera Italiana, \\ Scuola Universitaria di Musica, Lugano, Switzerland \\ NICHOLAS J. BAILEY \\ School of Science \& Engineering, University of Glasgow, UK
}

\begin{abstract}
Research has indicated that the magnitude of physical expressive movements during a performance helps to communicate a musician's affective intent. However, the underlying function of these performance gestures remains unclear. Nine highly skilled solo pianists are examined here to investigate the effect of structural interpretation on performance motion patterns. Following previous findings that these performers generate repeated patterns of motion through overall upper-body movements corresponding to phrasing structure, this study now investigates the particular shapes traced by these movements. Through this we identify universal and idiosyncratic features within the shapes of motion patterns generated by these performers. Gestural shapes are examined for performances of Chopin's explicitly structured A major Prelude (Op. 28, No. 7) and are related to individual interpretations of the more complex phrasing structure of Chopin's B minor Prelude (Op. 28, No. 6). Findings reveal a universal general embodiment of phrasing structure and other higherlevel structural features of the music. The physical makeup of this embodiment, however, is particular to both the performer and the piece being performed. Examining the link between performers' movements and interpreted structure strengthens understanding of the connection between body and instrument, furthering awareness of the relations between cognitive interpretation and physical expression of structure within music performance.
\end{abstract}

Submitted 2013 January 15; accepted 2013 June 27.

KEYWORDS: performance motion, phrasing, gesture shapes, structural interpretation

\section{INTRODUCTION}

STUDIES are increasingly finding that musicians' body-movements offer substantial information about the cognitive, emotive and communicative processes involved in performing music and that these non-soundproducing movements are of considerable importance to an audience's experience, interpretation and understanding of the music performed (Palmer, 1997; Davidson, 2007; Jensenius, Wanderley, Godøy, \& Leman, 2010; Dahl, Bevilacqua, Bresin, Clayton, Leante, Poggi, \& Rasamimanana, 2010). In this study we examine performance motions in relation to musical structure, particularly phrasing structure, in order to investigate whether performed gestural shapes indicate structural features of the composed music consistently within pieces and across performers. A quantitative method for the representation and analysis of performance movement is applied here to assess the impact of performers' individual interpretations of structural segmentation on the shape and form of their performance gestures.

It has been suggested that performance gestures occur on multiple levels, representing technical, expressive and communicative cognitive functions. Gestures include, but are not restricted to: movements directly necessary for the generation of sound; movements used to facilitate sound production, which affect 
the tone or feel of the sound without actually producing it; and movements that are supplementary or separated from sound production, existing as ancillary or concurrent motions not directly involved in sound production yet are arguably as important as those that generate the sounds themselves (see Cadoz \& Wanderley, 2000 and Jensenius et al., 2010 for review). The focus of this investigation is on the function of those non-sound-producing, non-technical concurrent motions.

\section{The expressive role of non-technical movements}

Although referred to as ancillary movements (Cadoz \& Wanderley, 2000; Jensenius et al., 2010), due to the fact that they are non-sound-producing or -facilitating, these non-technical motions are not of little importance, as the term ancillary implies. As observers of a live performance, we derive meaning from the audiovisual combination of technical and non-technical performance movements. When considering the meaning conveyed audibly by the performer alongside these non-sound-producing movements and the audiovisual experience of the observer, these movements become anything but ancillary. It has been suggested that changes in non-technical body motion aid communication with co-performers (Keller \& Appel, 2010; King \& Ginsborg; Goebl \& Palmer 2009; Clayton, 2007), are involved in memory storage and retrieval (Munoz, 2007; Godøy, 2010), and can display intended expressive (Davidson, 1993; Thompson \& Luck, 2008; Clarke \& Davidson, 1998) and structural qualities of a performance (Vines 2006), influencing audience interpretations of these expressed qualities (Davidson, 1994; Broughton \& Stevens, 2006; Dahl \& Friberg, 2007; Nusseck \& Wanderley, 2009). As a result, rather than being considered as ancillary, such performance motions will be referred to here as non-technical (not related to the production or modulation of sound[2]) or concurrent gestures, describing observed body motion that occurs alongside technical and facilitating movements that, although non-sound-affecting, may be considered meaningful and repetitive.

Non-technical performance movements, while notably reduced, have been seen to persist when a performer alters their intended expressivity from exaggerated to restricted or deadpan (Davidson, 1993; Thompson \& Luck, 2008). For pianists and violinists Davidson (1993) showed that the level of intended expressivity is related to the level of a performer's non-technical body motion. Performers altered the amount of body movement according to whether their performance was intended to be deadpan, expressive or exaggerated. Although the detailed relationship of performance motion and structural intention was not studied, Davidson's research indicates that it may be the magnitude and corporeal location of the motion that conveys expressivity, and not the motions' shape or regularity of occurrence.

There have been various suggestions as to the cause and function of non-technical performance gestures. Patterns of motion have been seen to persist throughout repeated performances by the same musician (Wanderley, 2002), with individuals finding playing uncomfortable when asked to restrain or restrict these movements (Wanderley, Vines, Middleton, McKay, \& Hatch, 2005). Wanderley and colleagues also noted that some movements corresponding to structural events within the musical excerpts were similar among different performers while other movements remained particular to an individual (Wanderley, 2002; Wanderley et al., 2005). It is not clear from these studies, however, whether there were causal relationships between structural events evoking patterns of motion (regardless of whether the location in the body of these movements and their shapes were generalisable or individualised across performers) and a performer's individual performance style, interpretation or expertise.

These non-technical or concurrent movements are often repeated and appear meaningful not only to performers but also to their audience and co-performers (Dahl et al., 2010; Jensenius et al., 2010; Platz \& Kopiez, 2012). Movement patterns have been seen to refer to more than simply the degree of intended emotion and expressivity persisting when little to no expression is intended, suggesting that the presence of such movements may be related to some other feature of music performance, such as the expression of structural features, memory retrieval or experience and expertise. Studies of relationships between nontechnical performance movements and the structural features of scores have yielded conflicting results, identifying differing body regions as central to the movement observed (Clarke \& Davidson, 1998; Davidson, 2007). Motions of the head and torso have been identified as meaningful in piano performance, relating to meter and rhythm as well as structural intent. Davidson (2007) indicated that movement shapes of the head and upper torso were recurrent for expressive locations in the score previously identified as salient by six independent judges. Davidson describes these repeated motion shapes as arising from the performer's expressive 'centre of moment', the physical core from which expressive information about the performance is extended and dispersed to other regions of the body (Davidson, 2007, p. 386). This is based 
upon theories of dispersal of locomotion by Cutting, Proffitt, and Kozlowski (1978), who demonstrated that any part of the motion cycle of walkers could reveal similar information regarding the target expression (in these instances the recognition of a walker's identity, whereas Davidson applies this theory to levels of expression within performance). In examining the relationship of head motion to phrasing structure, Camurri (2004) found that the temporal patterning of a solo pianist's head motion did not match the twobar phrasing structure of the Scriabin Étude they were performing. By contrast, Thompson \& Luck (2008, 2011) found that the head movements of their pianists did appear to follow the phrasing structure of the pieces performed. Similar findings have been reported for clarinettists, with performers' non-sound related movements, such as bending at the knees and waist and tilting or circling of the clarinet bell, appearing to correspond to features within the music's structure (Wanderley et al., 2005). Due to a lack of comparative repeated measures within analyses or between methods the extent to which such motions are responses to musical structure or are matters of individual performance style remains unclear.

Investigations into the communicative role of these non-technical movements have indicated that not only can an audience infer the intended mood and character of a performance and make social judgements about a performer's expertise, but they are also capable of inferring complex structural relations and segmentations from performance motion alone (Platz \& Kopiez, 2012). This implies a relationship between the perception of structural features and their expression by a performer (Palmer, 1989), although the influence of interpretation and intended expression has yet to be considered with regard to visible non-technical performance motion. Palmer (1989) demonstrated that performers' specific interpretations of structure effected audible characteristics of their performance, such as melody lead and expressive timing. This is extended by Rink's (2002) assertion that interpretation of a score influences expressed performance style. We propose that there will be a similar effect to those noted for the audible aspect of performance evident within a performer's non-technical performance motions, particularly with respect to phrase structure.

\section{Identification of performance gesture}

Following theoretical approaches to gesture categorisation in speech and social interaction, such as those by Kendon (2004), McNeill (2005) and Goldin-Meadow (1999), several proposals have been made for the detection and identification of instrumentalists' performance gestures, leading to taxonomies of the nuances exhibited by individual performers (Davidson, 2007). These describe actions such as nodding and shaking the head, leaning and bending the body, flicking and circling of the wrists and bending of the knees at salient events within a performance. However, the precise nature and shape of these movements has not previously been fully examined, nor has any consistency been observed in this regard between different performers or performances.

Investigations into particular music-related shapes have concentrated on using Becking curves to analyse patterns of movements made by performers, audience members and conductors. First analysed and published by Sievers (1924), the movements made by highly experienced conductors using lighted batons in response to various classical works were classified in qualitative, observational studies (Sievers, 1924; Repp, 1993). The pattern traced by the baton connects "points of metric gravity that vary in relative weight", which were thought to represent a composers' written intentions. These notated intentions are represented in the conductors' movements, projected through performers' audible expressions, and would then be reflected within a listener's music-induced movement patterns (Repp, 1993).[3] Van Noorden (2010) presents a review of research offering a more quantitative approach to the recording and representation of performance gestures, including those using samba dancers (Nevada \& Leman, 2007) and studies of participants' ability to express synchrony in time to a marching fragment using extended joysticks (Styns \& Van Noorden, 2006). However, gesture patterns are identified from visual observation and subjective analysis of audiovisual-recorded stimuli, and the authors themselves note a need to refine methodology in order to qualify results. That said, the patterns arising from the samba dancers' hand and foot motion imply that the dancers embody different metrical levels of the music within different body regions. Although a useful tool for the investigation of music-induced movement, the assumptions of predefined motion patterns may sometimes mask the retrieval of gestures as they are produced in real time. To counter this, the current paper concentrates on mapping the actual route of performance motion to reveal underlying patterns that can later be applied to gesture-identification and segmentation models.

Applications of segmentation models for the detection and parsing of performance motions have recently been proposed by Desmet, Nijs, Demey, Lesaffre, Martens, and Leman (2012), Caramiaux, 
Wanderley, and Bevilacqua (2012), and Rasamimanana (2012) to overcome a lack of recognised types and shapes of gestures within music research, with a view to computational modelling and categorisation of performance-gesture types. In the examination of a solo clarinettist's performance, Desmet and colleagues (2012) assess the relationship between the performer's expressive body movements and certain structural features within the score identified by the performer as being focal points of intended expression. Using the performance of a musical excerpt unfamiliar to the performer, and through the temporal modelling of intended targets and motion acceleration, Desmet et al. (2012) found that a relation did exist between the cognitive focus of their performer on musical targets and exhibited body movement.

Caramiaux et al. (2012) apply segmentational hidden markov models (SHMM) to the angular trajectories of four clarinettists' bell movement during repeated performances of Brahms's First Clarinet Sonata (Op.120). This was to determine the shapes of performance motions in relation to different time structures within the piece. Their study is based upon the earlier assertion by Wanderley and colleagues (Wanderley, 2002; Wanderley et al., 2005) that clarinettists' expressive intentions may be characterised by the degree of bell movement within a performance. Caramiaux and colleagues' model assumes a dictionary of primitive base shapes, with their analysis determining that performers exhibited a tendency to form circular shapes with the clarinet bell relative to structural segmentations. Sequences of shorter motions were seen to occur at phrasing boundaries, with longer, smoother base-shapes being formed throughout phrases. Patterns of motion were seen to be repeated by performers across subsequent performances, with the direction of circular motion varying between performers while the timing and length of circular gestures appeared structurally consistent. Although the dictionary of base shapes offers an exhaustive range of motion possibilities, the proposed model appears to consider the direction of motion between beginning and end positions of movement patterns as equivalent (parameters appear limited in the ABBA vector equivalence). As a result the identified shapes of motion do not appear to consider the actual route of the motion vector, potentially masking significant repetitions and trajectories.

Rasamimanana (2012) proposes a comprehensive approach to the modelling and parsing of violinists' continuous expressive gestures during performance based upon hierarchical hidden markov models (HHMM). This model is based upon the physiological, experiential and instrumental constraints placed upon a performer in order to determine the shape of exhibited performance gestures. Rasamimanana describes the motions as falling within a performer's 'space of possibilities' (SoP). The study focuses only on those actions technically necessary for the production of sound, with the proviso that these motions are central to definitions of actions in interaction with electronic sounds. The proposal to use actual observed motion patterns for the modelling and analysis of performance gesture, rather than a set of assumptions concerning predetermined base shapes, appears highly successful, with model parameters capable of adjusting to fit isolated motion patterns of similar shapes but of different forms within the input performance. As a result the HHMM seems to identify instances of repeated gesture patterns within a particular performance by a given performer quite accurately.

Despite such proposals for the segmentation and parsing of performance gestures, no studies to date have quantifiably linked any particular motion patterns to intended or notated structural and emotive expressions of meaning across multiple music performances (Platz \& Kopiez, 2012). Before a model may be effectively applied to different motion sequences, it is important to discover the form such gestural patterns may take so as to remain objective in gesture detection and analysis, reducing the risk of empirically over- or under-estimating the relevance and size of meaningful patterns. It is the premise of this article that before performance gestures may be successfully and consistently computationally detected, they must first be identified over multiple instances, performers and performances as being repeatedly produced and conceptually meaningful.

The authors have previously identified motion patterns as recurrent and idiosyncratic within nine highly skilled performers' individual motion profiles, generated through principal component analysis (PCA) of overall upper-body and head performance motions (MacRitchie, Buck \& Bailey, 2013). A motion profile, as defined in this previous paper, is a weighted combination of the first few principal components such that the total variance explained is above $90 \%$. These motion profiles are taken as a $2 \mathrm{D}$ representation of the movement that best describes the overall patterns of motion described over the 28 markers in threedimensional space. The motion profiles produced across the group of pianists indicated globalised variations of motion size and direction that fluctuate repeatedly and consistently in accordance with performed phrasing structure for Chopin's Prelude in A major. This article extends previous research, examining the performances and interpretations of Chopin's Prelude in B minor and comparing these back to performances of the stricter rhythmic structure identified by performers within the A major Prelude. 
It is hypothesised that the nature and form of these shapes, traced through non-technical, concurrent performance motions, will be related to each performer's intended expression of structural hierarchy as they interpret it within the compositions. The following sections demonstrate, through the use of polar coordinate projections, the visible shapes traced by performers' head and body motions around their central gravitational pole. These are presented as spherical analyses of motion. Repeated patterns of movements corresponding to phrasing structure can be termed as phrasing gestures as they have been seen to be meaningful both in their temporal generation and consistency as well as in their ability to convey meaning to an observing audience (for discussion see Buck, 2013). The patterns elucidated by means of PCA and spherical analyses will be assessed here with respect to the hypothesis that structural performance gestures may be highly individualised and potentially dependent on a performer's personalised style and interpretation.

\section{METHOD \& DESIGN}

For full discussions of the methodology and recording procedures employed for three-dimensional motion capture, visual and auditory recordings of the performers presented within this study, we would direct readers to the following previous publications: MacRitchie (2011); MacRitchie et al. (2013), Buck (2013). Previous investigations sought to examine the generation of repetitive motion over phrases of similar rhythmic duration and complexity by comparing the two-bar phrasing structure of the A major Prelude, and similar instances arising in the B minor Prelude, in order to investigate consistency of performance gesture over similar phrases within multiple performances of differing compositions (MacRitchie et al., 2013). Those findings are extended here to consider the more varied phrasing structure of the B minor Prelude in its entirety. This allows us to assess the impact that different performers' interpretations of structural segmentation may have on individual gestural shaping of phrases with similar or varying rhythmical makeup. Performers' self-reported structural intentions and interpretations, obtained during post-performance interview, are combined with previous and novel analyses of performance motion to examine the relationship between cognitive intent, evidenced through their interpretation, and physical expressions of performance motion. As a result, the following provides only a summary of the empirical methodology used, with added descriptions of the extensions made to previous investigations.

\section{Participants, Apparatus \& Design}

Nine highly trained solo pianists, each with a minimum of 10 years performance experience, were paid a one-off sum of $£ 25$ for their participation. Each pianist performed two selected Chopin Preludes (Op. 28, Nos. 7 \& 6) on a Roland RD-150 weighted keyboard[4] and was recorded using a 12-camera infrared Vicon 3D-motion capture system.[5] Twenty-four retro-reflective markers were attached to performers' upper bodies fitting the model described by Cutti, Paolini, Troncossi, Cappello, and Davalli (2005), to obtain detailed upper-body performance motion recordings. Four additional markers were positioned on the head to include these movements within the data (see Figure 1). Scores for the selected Preludes were obtained from mutopiaproject.org and provided to pianists one month prior to recording. This ensured that all performers began with the same notated directions regarding tempo and dynamic instruction. Score examples are provided in Appendix A.

Performers were instructed to play the selected pieces as they would in a typical live performance scenario. No other instructions regarding performance style were issued to any of the participants. Performers were applauded into the recording room when ready so as to maintain the feeling of a live performance setting. The two selected pieces were played as part of a three-piece programme (comprising Chopin's Preludes in A major, B minor and E minor, Op. 28, Nos. 7, 6, \& 4 respectively) in the order of their choosing. Those pianists who wished to perform with the aid of the scores were able to do so, as all nine performers were capable of performing the pieces from memory and so were considered to have formed concrete mental representations of their interpretations within their performances. 


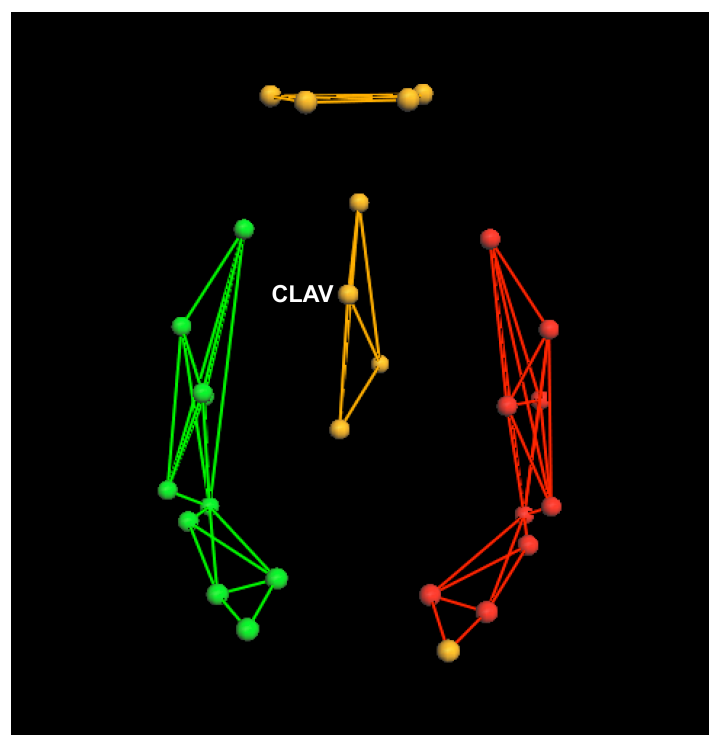

Fig. 1. Marker placement model for upper-body 3D-motion capture. Trajectories of all 28 markers, depicted (represented by circular points positioned at key musculature and joint locations across the performers' torso, head and arms, were used for the calculation of PCA motion profiles. Spherical analyses demonstrate movement patterns of the head and torso regions. These are depicted centrally in the above figure and represented in orange/a lighter hue. Motion of the clavicle marker was also examined to consider respiratory effects. This marker (CLAV) is positioned at the top of the sternum, just below the centre of the clavicle bones of each performer.

\section{Selected Preludes}

Examples of the full score for Chopin's Prelude in B minor issued to participating pianists and the opening phrase of the Prelude in A major are provided in the Appendix A. Performers' structural interpretations of phrasing were in agreement for the eight two-bar phrases of the A major Prelude and for the opening five phrases of the B minor Prelude, although some performers did group these phrases into different higherorder structures. Also depicted in Appendix A, Figure $b$, are indicators representing phrasing boundaries as taken from theoretical analyses. All reference to phrasing segmentation for the two preludes will relate to these markings as depicted unless stated as otherwise within the text. For more detail on the precise nature of phrasing for the A major Prelude see MacRitchie et al. (2013).

Chopin's Prelude in A major was selected for performance analysis to discern whether performers do make repeated phrasing gestures across analogous phrases (shown previously in MacRitchie et al., 2013) and to identify the shapes of any repeated gestures or movement patterns observed. Chopin's Prelude in B minor was selected to further examine movement patterns across the two pieces by multiple performers. This extends analyses of the A major Prelude performances to consider movements generated during phrases varying not only in structural importance but also in duration, rhythm, pitch and harmony.

The B minor Prelude begins with a two-bar motif that is repeated several times throughout the 26bar piece. The first eight bars of the piece can be considered as the first section of the piece (or the antecedent phrase -Dodson, 2011), which includes repetitions of this initial two-bar motif, altering in pitch, with the second repetition extending into a longer phrase duration. Of note is the entry in the second half of this longer phrase of a right-hand (RH) auxiliary melody (Rink, 2012). The second section of the piece (or the consequent phrase, as marked out by Dodson, 2011) begins with a strict repetition of the opening material, the consequent repetition here changing in harmony to bring us towards the $\mathrm{C}$ major modulation that stretches until the end of bar 14. This is considered by Dodson (2011) to constitute a written out fermata. At bar 15 we notice the re-entry of the auxiliary melody previously heard in the RH at bar 7 . This time the melody continues for 4 bars ending on a $\mathrm{G}$ major chord. The auxiliary melody is repeated again in bars 19-22, this time finishing on the tonic, with the last 4 bars acting as a small coda. When examining different first editions of this piece (available via www.ocve.org.uk) one finds differences in the phrase markings at bars 15-19. In the French and English first editions, along with a select number of later 
editions, bar 15 is phrased as being separate from the next three bars. However, when this material is repeated in bars 19-22 it is marked as one four-bar phrase. In other editions, such as the German first edition and the score provided to the participating pianists, these two sets of four bars $(15-18,19-22)$ are phrased identically, as two four-bar phrases (see Appendix A, Figure $b$ ).

\section{Post-Performance Interviews}

Each pianist was interviewed after their performances with respect to their music-analytical interpretations of the selected pieces and their views regarding the role of movement within music performance. Performers were presented with the audio-visual recordings of their own performances and ask to mark on the scores their intentions with regard to structural segmentation of phrasing. A short semi-structured interview was conducted to ask performers whether they viewed movement as having a general purpose for any or all performers and were then asked to describe any particular movements they themselves had made and whether these were intended to convey particular information, such as phrasing, or to facilitate or emphasise audible aspects of their performance.

\section{RESULTS \& OBSERVATIONS}
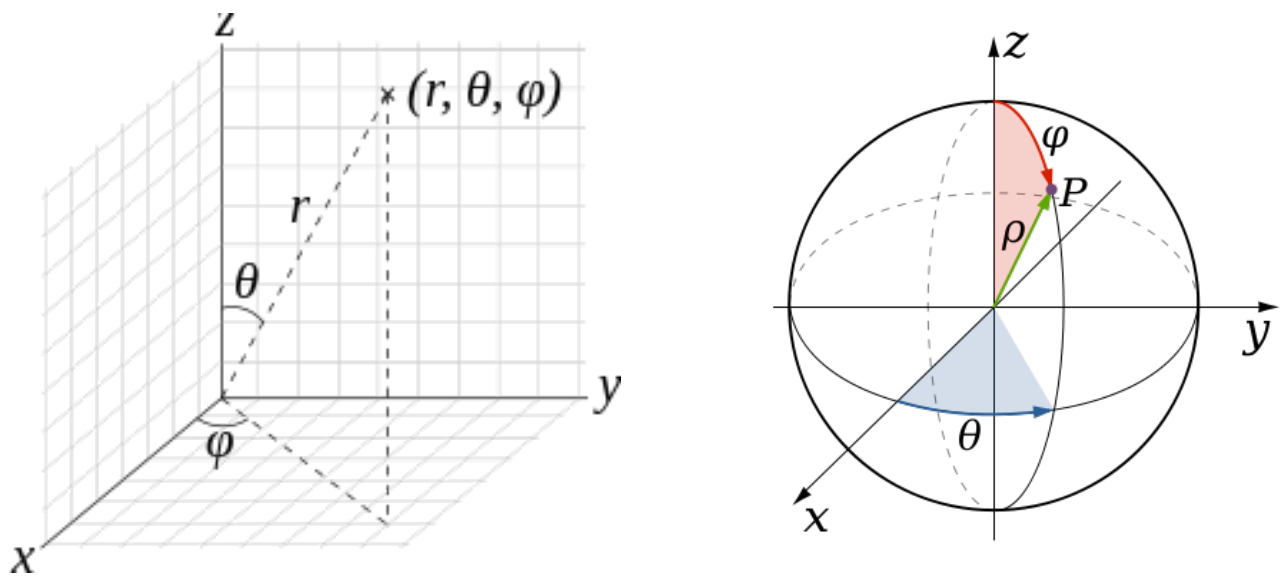

2a)

2b)

Fig. 2. A depiction of how spherical, or polar, coordinates translate into traditional Cartesian 3-dimensional space. The asterisk denotes the location of the coordinate, with rho (typically ' $\rho$ ' but also represented as ' $r$ ' above in Fig. 2a) representing the magnitude of vector being examined, in this case motion. Theta $(\theta)$ and phi ( ) then describe the angulature of the vector relative to its original position or central gravitational pole. Theta describes the variation of vertical angle within the z-dimension, for example referring to the tilt or inclination of a performer's torso or head. Phi then describes the rotation of the object within the horizontal plane, also referred to as the azimuth angle.

(Images have been reproduced from Wikimedia Commons under the public domain licence use. 2a: Andeggs / Wikimedia Commons / Public Domain. 2b: Inductiveload / Wikimedia Commons / Public Domain).

Spherical analyses were conducted for all nine pianists' performances of the A major Prelude to identify whether performers make similar use of gesture shapes and patterns when they play a score. Motion coordinates were normalised so that Cartesian $(\mathrm{x}, \mathrm{y}, \mathrm{z})(0,0,0)$ represented the coordinates at the start time of each performance. Each ordinate was then normalised to the range of its own axis, offsetting the data so that point 0 reflects the centre of motion for each performer. All data coordinates maintained their original distance from one another and the shape of motion was retained. The result, therefore, is to extend the event horizon to reveal the true shape of the motion in spherical space (as depicted in Figures $2 \mathrm{a} \& \mathrm{~b}$ ). These are compared to the motion profiles of MacRitchie et al. (2013) to further explore the comprehensive and integrated patterning of performance motion made by these performers. In doing so we explore the local 
movement of these regions in relation to the global movement patterns of the upper-body, examining the interdependence of the 28 measured markers and their 3-dimensional trajectories. Body movements are then assessed for performances of the B minor Prelude to provide deeper investigation into the effects of varying compositional structure and complexity, and into the influence individual interpretations of structure have upon each musician's performance motion.

\section{Identifying Motion Patterns: A major Prelude}

\section{PCA MOTION PROFILES}

PCA motion profiles reveal that all nine performers exhibit repetitive changes in the magnitude of global marker displacement corresponding to the eight two-bar phrases of the A major prelude (Appendix B).[6] A two-way ANOVA was performed for factors of Phrase Number and Performer to examine the variance in the placement of local maxima for each phrase by the nine performers. It revealed a significant main effect of Performer $(F[8,56]=15.06, p<0.001)$ but no significant effect of Phrase Number. These results indicate that while repeated patterns of motion significantly relate to phrasing segmentations for all performers, the temporal placement of the maxima is not related to the hierarchical importance of individual phrases: all performers are consistent across phrases in the location of their individual motion maxima. Closer inspection of the mean positioning of phrasing segmentations within the significant main effect of Performer suggested that performers were idiosyncratic in their temporal location of movement maxima. To test this hypothesis multiple comparisons were conducted a priori using Bonferroni adjusted alpha levels. Results indicated that performers varied between one another in their overall pattern of phrase positioning. Tests of homogeneity confirm that the variance within each factor level assessed (i.e. each performer) is equal, with no significant homogenous subsets arising. Mean positioning of phrase boundaries varies significantly between performers, with post-hoc comparisons indicating that each of the 9 performers differs from at least 2 and at most 5 performers in the set in their mean location of phrasing (the distance of motion maxima or minima from actual phrase boundaries),[7] i.e. in 16:36 comparisons, with additional comparisons approaching trend significance. Therefore, some performers locate maximum motion variance before the phrasing boundary, while others emphasise phrase transitions in the time period after the actual boundary had occurred, and each does this to varying degrees. While performers differed between one another in their placement of the maxima, each was consistent in their individual use of motion relative to phrasing across the eight phrases; for example, those who indicated phrasing before the boundary of phrase 1 were seen to continue this pattern in $\geq 75 \%$ of phrases (at least $6: 8$ instances) and vice versa (i.e. where motion emphases are located after the actual phrase boundary for phrase 1). All performers' motion profiles reveal points of global maxima and minima occurring at structurally salient events. While individual performers expressed these events differently, all exhibited the point of greatest variance in motion profile magnitude at sectional boundaries (ends of phrases 4 and 8 ) or at the occurrence of the harmonic climax within the piece (phrase 6, time period 0.7-0.8).

As each performer's motion profile is made up of different combinations of single markers, depending on the specific location in the body of their largest variances in movement, the coordinate spaces of salient features within motion profiles are not directly comparable. The variance of profile magnitude represents an overall measure of motion variance and positional change across multiple markers and spatial dimensions. Therefore, a minimum in one profile is not necessarily directly comparable with a minimum in another. What is important here is that profiles demonstrate that all performers make repeated patterns of motion variance in line with phrasing segmentation. Additionally, their largest points of global motion variance occur at time-points corresponding with salient structural features within the Prelude, such as at the harmonic climax of bar 12, sectional boundaries, or the cadential close of the eighth phrase.[7] While motion profiles may first appear similar in their fluctuations of the magnitude of variance, no two are the same with respect to contributing markers or the directionality of displacement of these markers, which combined to form the overall motion profiles for each performer.

\section{SPHERICAL ANALYSES:}

Having found no specific leading markers in the PCA motion profiles, performers' head and torso movements were examined relative to phrasing to investigate claims within the literature that these regions hold information specifically relating to rhythm and structural segmentation. Spherical (or polar) 
coordinates referencing the changing locations of markers within these regions were calculated over time to show performers' spherical performance motion patterns relative to their individual central gravitational poles. This was achieved by normalising the Cartesian data points within each of the ordinates' dimensional ranges and calculating spherical coordinates, rho, theta and phi, using the following calculations (see Figures $2 \mathrm{a} \& 2 \mathrm{~b})$ :

$$
\begin{aligned}
& \text { Rho }=\wp\left(x^{2}+y^{2}+z^{2}\right) \\
& \text { Theta }=\arccos (z-r) \\
& \text { Phi }=\arctan (y / x)
\end{aligned}
$$

By transforming the coordinate space from Cartesian $(x, y, z)$ to Spherical $(r, \theta, \quad)$ it is often possible to reveal information that may otherwise be hidden beyond the event horizon. One of the novel benefits of using spherical coordinates to map performers' gestural movements is that it becomes possible to visualise the precise shapes of motion that were previously masked in Cartesian time-space. This enables recurring patterns of motion to become clear. The mapping of spherical coordinates relative to Cartesian 3dimensional space is presented in Figure 2.

Figure 3 demonstrates the spherical motion patterns for each of the nine performers' head motion throughout their A major Prelude performances. For brevity, spherical plots for three exemplar performers (Performers 2, $3 \& 9$ respectively) are presented in Figure 4, depicting the changing shapes of embodied structure across the head, clavicle (or upper sternum - see Fig. 1) and torso movements. While the progression of time itself is not indicated in the spherical mapping, the plots are intended to be an illustration of the shapes of repeated patterns arising within motion that have been identified through the motion profiles.[8] By comparing the patterns produced through spherical analyses (Figures $3 \& 4$ ) with the findings of the PCA motion analyses (Appendix B) it becomes evident that performers are making repeated shapes of motion relating to phrasing structure, and that these patterns or shapes of motion are present in varying forms throughout the different regions of the performers' body.

Each performer generates a pattern of motion particular to their individual performance style. This pattern, typically appearing as lemniscate $(\infty)$, annodated (S) or crescent (C) shaped movements, and less often appearing as cruciform $(\mathrm{t})$, sigmate $(\Sigma)$ and ' $\mathrm{L}$ ' shaped motions, is traced repeatedly by the given body region in accordance with each phrase. Individual performers are also seen to represent motion patterns differently across the three body regions examined. For example, Performer A's phrasing motion shows linear sweeping motions along the length of the keyboard in the torso motion, while generating more rounded motions with the head. In contrast, Performer B shows no change in the general shape of phrasing motions across body regions, instead demonstrating a broadening of the range and spatial location of pivotal points within the motion. Alternatively, Performer $\mathrm{C}$ shows a variation in the direction of phrasing gestures across the three body regions, with diagonal upwards motions of the head away from the keyboard at phrase endings varying from the parallel motions of the torso. Additional analyses have shown that performers' phrasing movements sometimes appear counter-productive to those movements required to produce sound (Buck, 2013). Performer $\mathrm{C}$ is an example of this, with the direction of head and torso motion appearing contrary to the direction of the performed melody.

Additional patterns are evident within the individual spherical plots (Figure 4), indicating variations in either the size (Performer A), end positioning (Performer B) or directionality (Performer C) of phrasing movements. Closer analysis of the time periods of these seemingly anomalous patterns reveals that performers are varying the shape, size or directionality (and often a combination of these) at points where higher-order structural features occur, such as the climax of bar 12 in phrase 6 . This is particularly evident in the additional loop to the right of the keyboard in the clavicle and head motion of Performer $\mathrm{C}$, for example (Figure 4, plot a), corresponding to her interpretation that this phrase is of particular importance compared to the preceding phrases. 

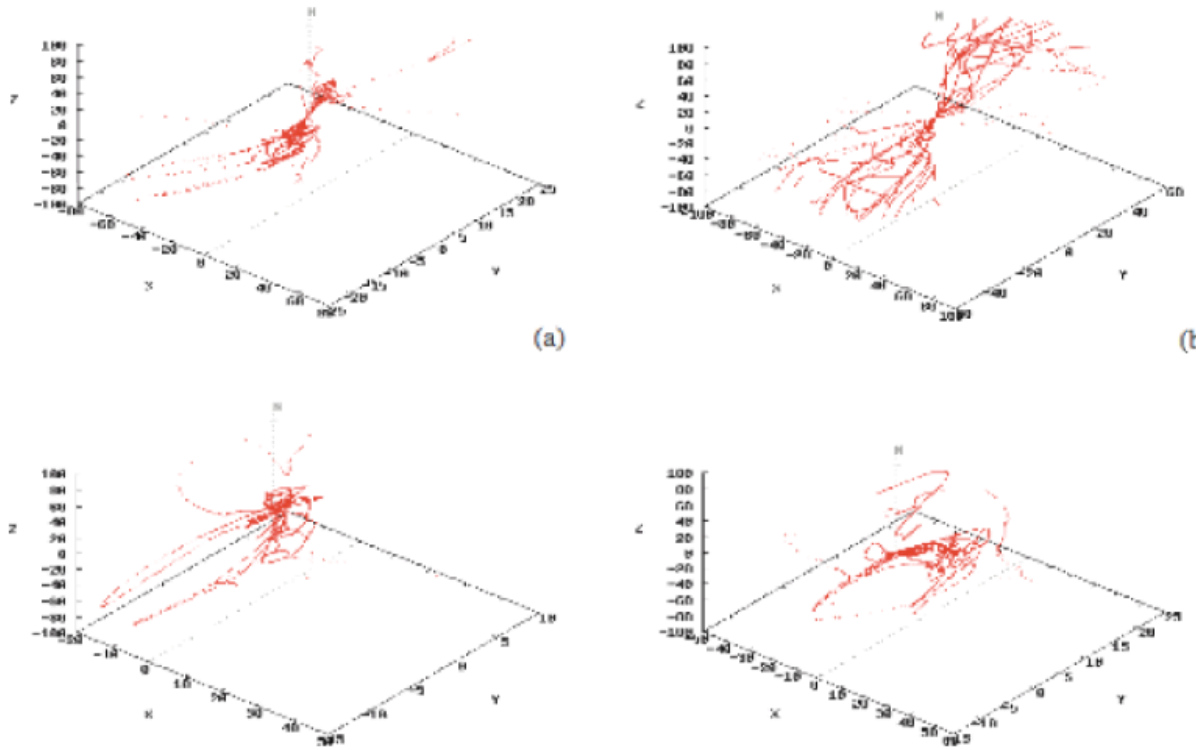

(d)

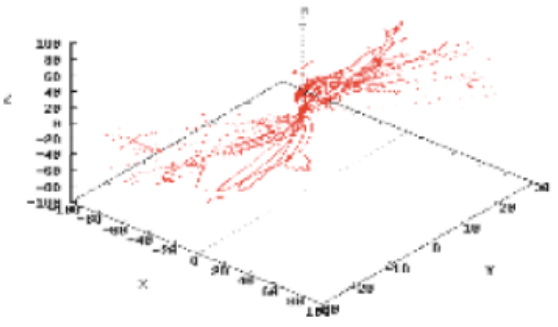

(g)

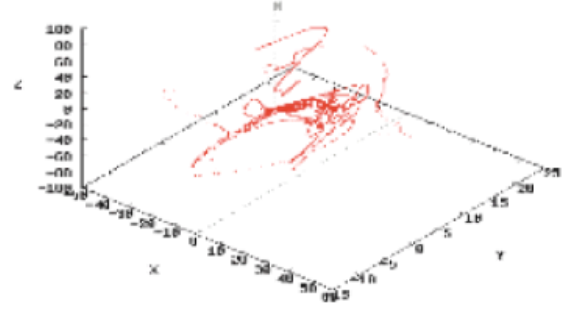

(e)

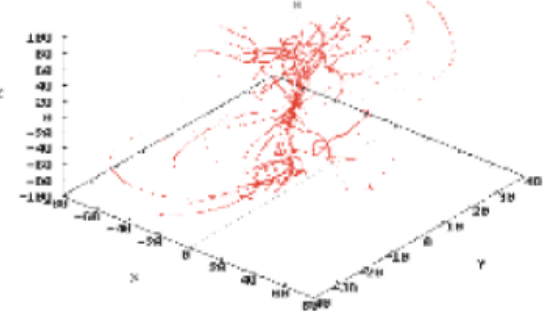

(h)

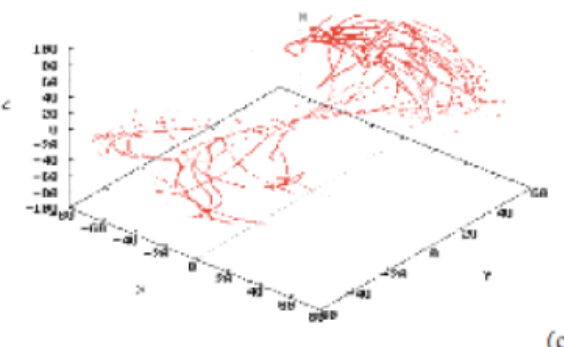

(c)

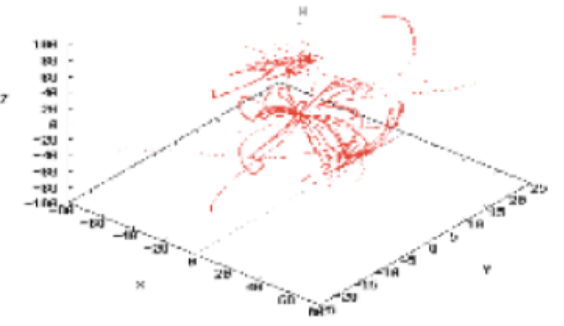

(f)

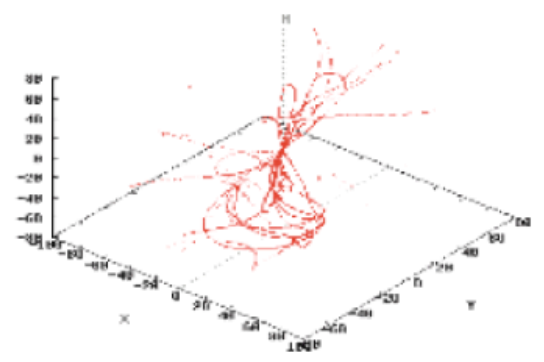

(i)

Fig. 3. Spherical analyses describing head motion of all nine performers during performances of the A major Prelude 

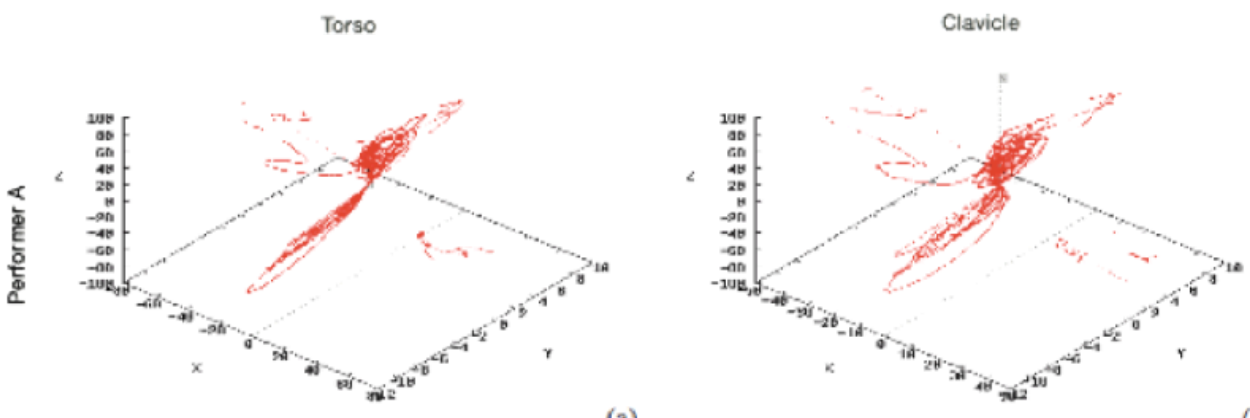

(a)
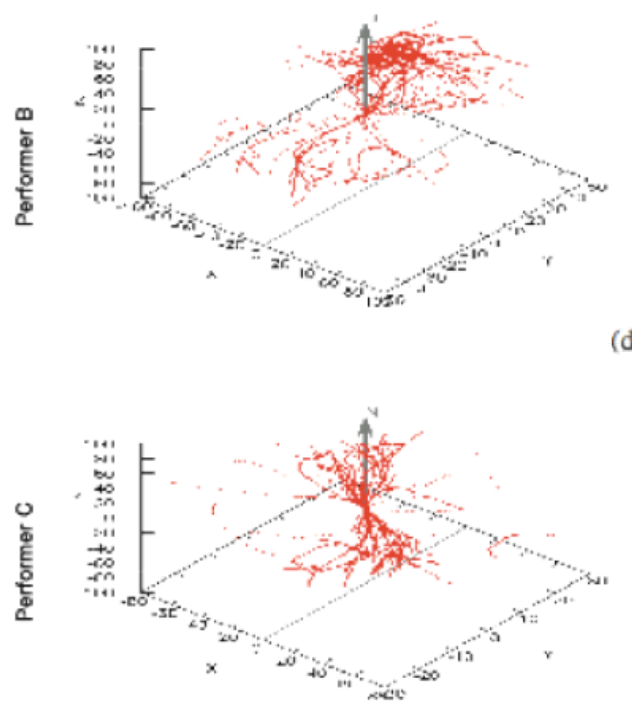

Fīgure X.X: (d)

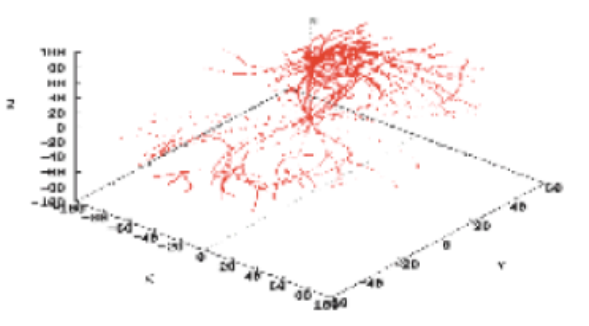

(e)

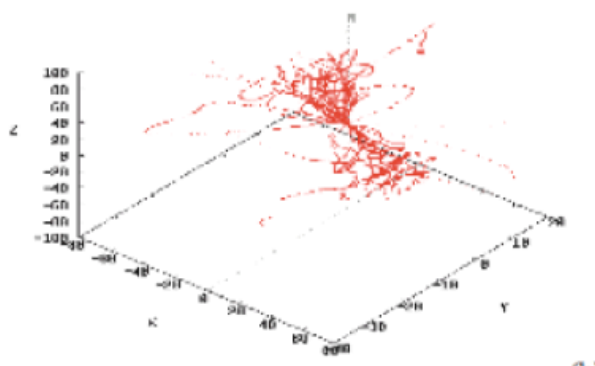

(g)

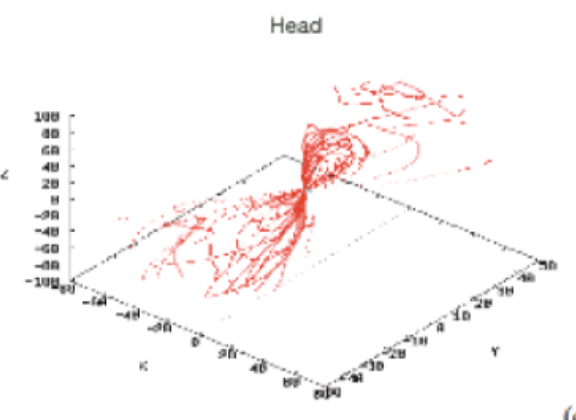

(c)

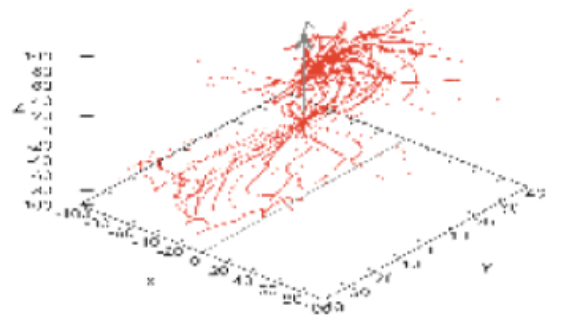

(f)

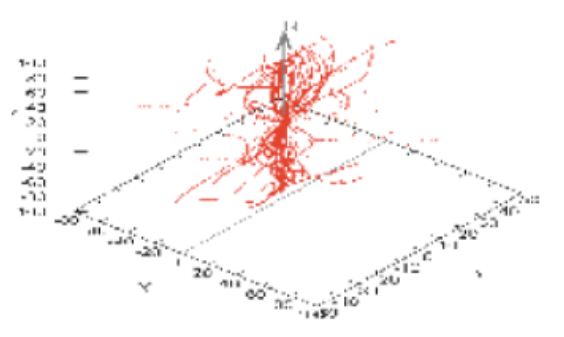

(h) (i)

Fig. 4. Spherical analyses for the head, clavicle and torso motion of Performers A, B \& C during performances of the A major Prelude (corresponding to motion profiles $b, c \& i$ in Appendix B). 


\section{DISCUSSION OF A MAJOR PRELUDE RESULTS}

Post-performance interviews indicate that all nine performers' structural interpretations agreed upon the eight two-bar phrases of the A major Prelude. Only two performers did not group these phrases into two 4phrase sections, 1-4 and 5-8. Instead, these two performers joined phrases 3 and 4 into a larger structural group within the first section.

Comparing performers' interpretations with results of the PCA motion profiles it is evident that the globalised body motion of each performer reflects their interpretations of the sub-phrasing, phrasing and sectioning within the piece. Motion profiles indicate, and spherical analyses confirm, that repeated patterns of motion are generated globally across the body in all three spatial dimensions. These repeated patterns of motion are highly individualised to each performer, with observed shapes being traced simultaneously by individual body regions. The shapes produced at different body regions differ for each performer, as does the manner in which they move the various regions of their body in time with phrasing. For some, the shapes generated are similar across different body regions, varying in the magnitude or direction of movement. For others different shapes are observed across different body regions. This altering of size, shape and direction of phrasing movements across body regions explains why no set markers or directions of marker displacement were found to be leading movement for any of the performers in the principal component analyses.

\section{Examining Impacts of Varying Interpretation: B minor Prelude}

\section{PCA MOTION PROFILES}

Previous research has presented analyses of the first five phrases of the B minor Prelude to support the findings of the A major Prelude that performers' movements vary in accordance to phrasing structure. This was due to performer agreement on the segmentation of the first five phrases of the B minor Prelude. To investigate the effect of structural interpretation on embodied action, motion profiles for the full performances of the B minor Prelude by all nine pianists are provided here in Figure 5. Analyses are informed by the intentions marked by each performer in the post-performance interview. As before, data has been time-normalised according to the timing benchmarks, indicated as vertical blue bars in Appendix A, Figure $b$. These benchmarks represent structural boundaries agreed upon by all nine pianists. Some performers further segment particular phrases into smaller motifs. These variations in structural interpretations by performers are investigated in the following analyses.

This is illustrated by the way performers approach the third phrase (bars 5-8). Performers 1 and 5 treat the four bars of this phrase as an extended version of the previous two-bar motif, as evidenced through their general motion. This extended motion corresponds with their score markings across these four bars. Performers 3, 8 and 9 also marked this four-bar block as one long extended phrase on the score. However, their general motion patterns show slight changes between this and the previous two-bar motifs, indicating a small variation within performance motions to take into account the latter part of the phrase. Performers 2 and 4 definitively separate this four-bar segment within their movement pattern into two two-bar segments corresponding with their markings on the right hand $(\mathrm{RH})$ melody of the score in bars 7 and 8 made during interview.

The $\mathrm{C}$ major modulation featuring small arpeggiated runs at bar 13/14, in what is marked as part of phrase 5 in Figure $b$ (Appendix A), is marked by nearly all performers ( 7 of 9 ) as being separate to the first part of the phrase. This may correspond to the suggestion by Dodson (2011) that these two bars are a written-out fermata. This division corresponds with their interpretations expressed during interview that this modulation is structurally separate from bars 11 and 12. This can be seen in Figure 4 as the juncture between two phrasing motions that occurs during time period 0.5 to 0.6 (phrase 5).

The re-entry of the auxiliary motif in the left hand at phrase 6 results in changes in motion patterns for all performers to accommodate this new material. Performer 1 marks phrases 6 and 7 identically as two long four-bar phrases and this is reflected in their motion profile, time period 0.6 to 0.8 . In contrast, although identical score markings are made by Performer 6, a different embodiment of structure is reflected in the motion profile. Although this performer marks phrase 6 as being one segment, within the motion profile the end of phrase 6 and the seventh phrase are concatenated by movements which appear to cross the phrase boundary. Similarly Performer 8 denotes phrases 6 and 7 as two blocks of four bars during post- 
performance interview, yet appears to split these phrases in half during their performance, with the end of phrase 6 and the beginning of phrase 7 forming one continuous gesture. Performer 3 also demonstrates this joining between phrases within their motion profile. However, for this particular performer, the first part of the movement, seen in phrase 6 , is mimicked in the latter part of phrase 7 , to a larger extent than is seen for Performers 6 and 8. Performers 5 and 9 both separate each of the sixth and seventh phrases into three subsegments in their movement patterns even though their marked interpretations indicate each should be one long phrase. Finally, Performers 2 and 4 both indicate that the first auxiliary motif in bar 15, repeated in bar 19, should be separate from the latter part of each respective phrase. This reveals itself in their motion profiles (more obviously for Performer 2) with each phrase appearing to have two distinct sections within the motion variance. Performer 2 also expresses this joining motion between the two phrases as seen for Performers 3, 8 and 9. For the final phrase, when comparing the motion profiles to the score markings made by performers, we see that differences in interpretation alter the shape of the continuous movement to some extent.

This relationship between a performer's score-marked intentions and the shapes seen in the motion profiles is not entirely straightforward. This may be a result of the method used to collect performers' intentions. Generalised markings of structural interpretations were requested rather than indepth detail of how individual phrases were treated by performers. The shape of the motion profile appears tied not only to the rhythmic makeup of each phrase, as evidenced by the change in shape between the first and second sections of the piece, but also to the performer's own interpretation of this. Interpretation appears to affect the boundaries and repetitions within certain aspects of expressed movements.

\section{SPHERICAL ANALYSES}

Having discussed how performers each make repetitive movements in their global upper-body motion that align with phrasing segments within the score, and having demonstrated that these are affected by performers' individual ideas on how phrases are segmented in the B minor prelude, spherical analyses allow us to examine the shape of these repeated movements and investigate how these patterns change throughout the upper body. Figure 6 presents spherical head motion for each of the nine pianists, represented as polar coordinates, rho (r), theta $(\theta)$ and phi ( ) along a 3-dimensional axis. The spherical analyses of all nine performers (Figure 6) are summarised in Figure 7 through examination of the three exemplar performers presented for the A major Prelude results. As before, spherical examination of changing horizontal ( ) and vertical $(\theta)$ angles with regard to radial displacement (r) of head, torso and clavicle motion throughout the performance, was focused on each marker's (or groups of markers') central gravitational pole.

Figure 6 clearly demonstrates the different ways in which individual performers embody phrasing structure. For all nine performers a repeated pattern of phrasing is evident in the trajectory traced by the head. As was observed for performances of the A major Prelude, individual patterns of repeated motion are seen typically to take the form of lemniscate $(\infty)$, annodated $(S)$, crescent $(C)$ and cruciform $(t / T)$ shapes traced by performers' head and body motion. While the patterns observed for the B minor Prelude appear to trace similar shapes for many performers (Performers 1, 2, 3, 7, 8 and 9; see Figure 3), the size and directionality of these shapes are not identical. Across performers and performances no two patterns are entirely the same, with trajectories varying in size, range and spatial direction. Quite different phrasing patterns are generated across the two pieces by Performers 4, 5, and 6 . These three performers can be seen respectively to change from generating sweeping,[9] crescent and lemniscate shaped patterns (see Figure 3, $d, e, f$ ) to sigmate $(\Sigma)$, crescent (following a rotated, almost inverted trajectory to that of the A major performance), and lateral ' $\mathrm{L}$ ' shaped patterns (see Figure 7, d,e,f). Variations in the generated shape and form of phrasing patterns demonstrate that one definitive pattern of motion signifying phrasing for all performers does not exist. This variation in the form of phrasing gestures was also observed for performances of the A major Prelude, with differences in patterns arising not only between performers but also within specific body regions of individual performers across the two preludes. While motion analyses show that all performers exhibit repeated patterns of motion relating to phrasing structure, spherical trajectories and motion profiles demonstrate that this embodiment of phrasing is highly personalised with different performers generating different patterns of phrasing not only between one another but also between performed pieces.

For performances of the B minor Prelude, the same variations in shape, style, direction and range can be seen for the three performers as were observed for the A major Prelude (Figure 7). However, when 
considering the different interpretations of this piece, there appear to be more variations that involve a changing of the pivotal range of motion for all three performers, i.e. the point where motion rotates to return toward the performer's central gravitational pole. This is particularly evident for Performer B, whose spherical motion can be seen to extend, curving further at the outer limits of the motion as the phrases progress. The point where motion patterns deviate from the typical phrasing pattern for each performer relate to points of climax within the composition. In this second Prelude, this is especially apparent for Performer A, whose torso and clavicle motion alters from typically producing sweeping movements along the keyboard for phrasing, to making circling movements down-and-toward, and up-and-away from the keyboard for phrase 6. Points where performers' spherical motion-patterns alter correspond to particular compositional features such as when the new auxiliary melody is introduced in the right hand of phrase 3, and when this is developed during phrases 6 and 7. These phrases also represent points of increased structural ambiguity, highlighting points within the piece where interpretations between performers vary. As a result it is plausible that performers may be altering their motion patterns to accentuate their intended structural expression during phrases with more varied analytical interpretations, such as phrases 3,5 and 6, to enhance their audience's understanding of that particular performance.

\section{DISCUSSION OF B MINOR PRELUDE RESULTS}

Post-performance interviews revealed that performers' interpretations differed at points of structural ambiguity within the B minor Prelude. These points included potential sub-phrasing within phrases 5 to 7 . Patterns of repeated motion evident within the PCA motion profiles reflected these differences of opinion, with patterns of globalised motion variance corresponding to individual performers' structural interpretations of phrasing segmentation and sectional grouping. Spherical analysis demonstrated that motion patterns adhere to temporal patterns of phrasing, as they appear within the performed audio stream [7], across the examined body regions. These findings are supported by the spherical analysis of the A major Prelude. The consistency of temporal patterning of movements throughout these regions goes a long way to explaining the absence of any leading markers within motion profiles. While the different regions of the body are often (but not universally) seen to trace different shapes or trajectories from one another, all of the movements created follow a timing pattern relative to performers' personal interpretations of phrasing structure. The shape, size and direction of these movements alter at points of key structural importance. In the case of the B minor performances, these points of gestural divergence also correspond to points of structural ambiguity between interpretations. It is plausible, then, that performers alter the shape (lemniscate etc.) and form (size and direction) of phrasing movements in order to draw attention to points of structural importance as well as to points where personal interpretation may vary. Changes in the spatial patterning of performance gestures within individual performances may then be seen to represent both expressions of individual interpretations of structure and performer individuality, as each performer is idiosyncratic in the temporal or spatial relation between their repetitive movements and their structural segmentation of the piece. 

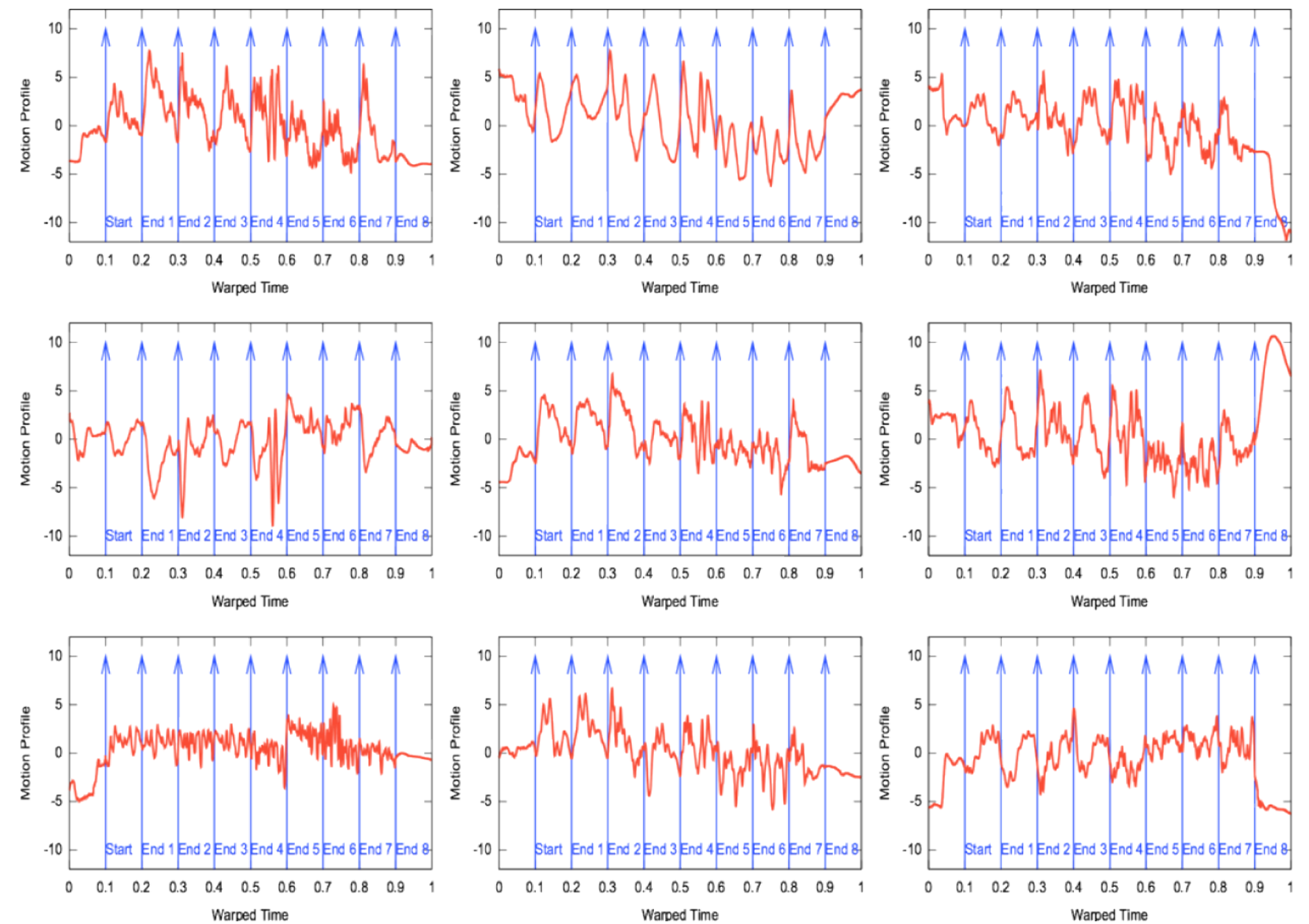

Fig. 5. PCA motion profiles for the nine performances of the B minor Prelude*. Individual motion profiles represent combinations of principal components explaining $>90 \%$ of performance movements exhibited by each of the nine performers (Performers 1-9 represented as plots $a-i$ in figure). (*Reproduced with permission from Musicae Scientiae). 


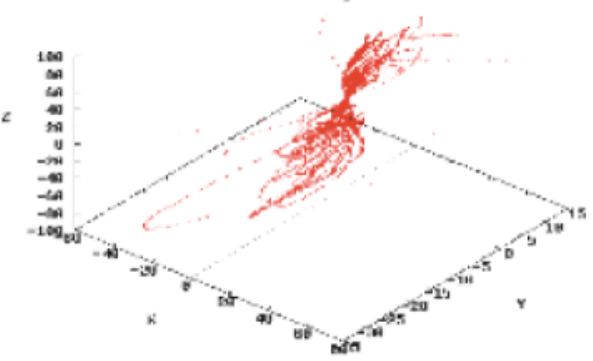

(a)

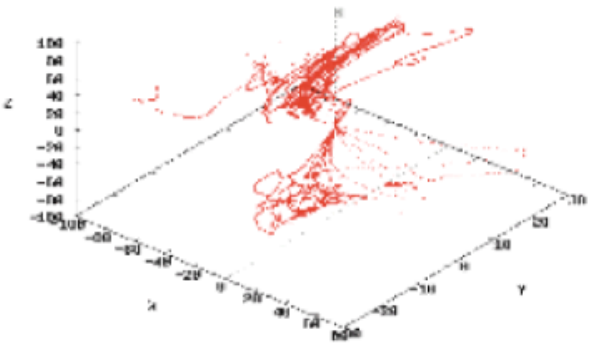

(d)

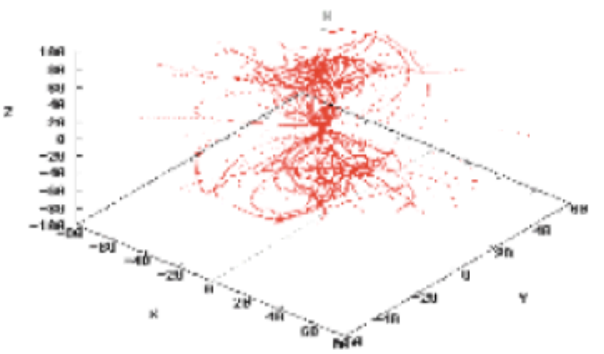

(g)

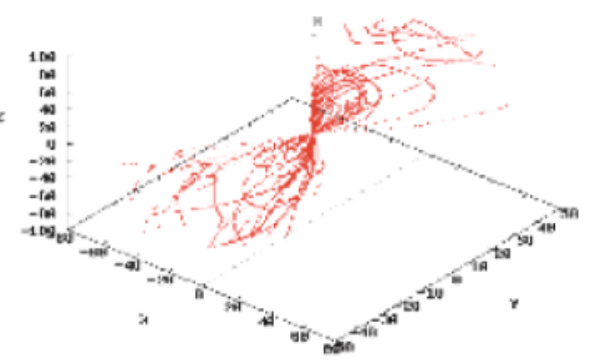

(b)

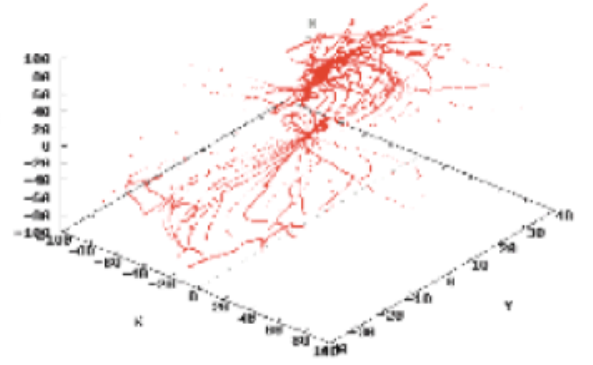

(c)
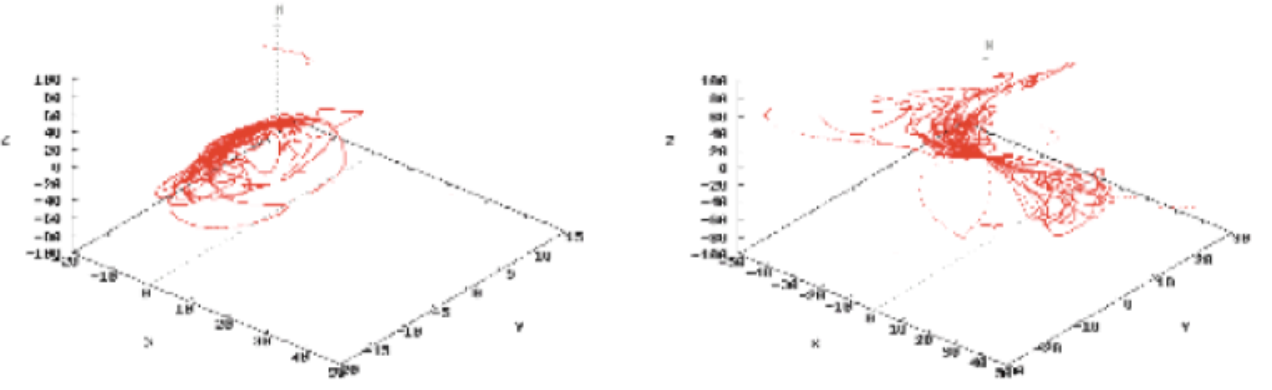

(e)
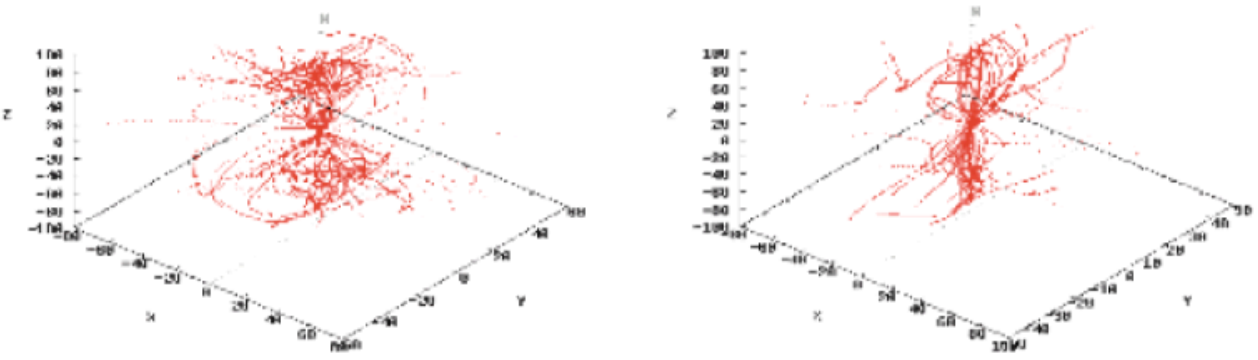

(h)

Fig. 6. Spherical analyses for the head motion of all nine performers during performances of the B minor Prelude. 

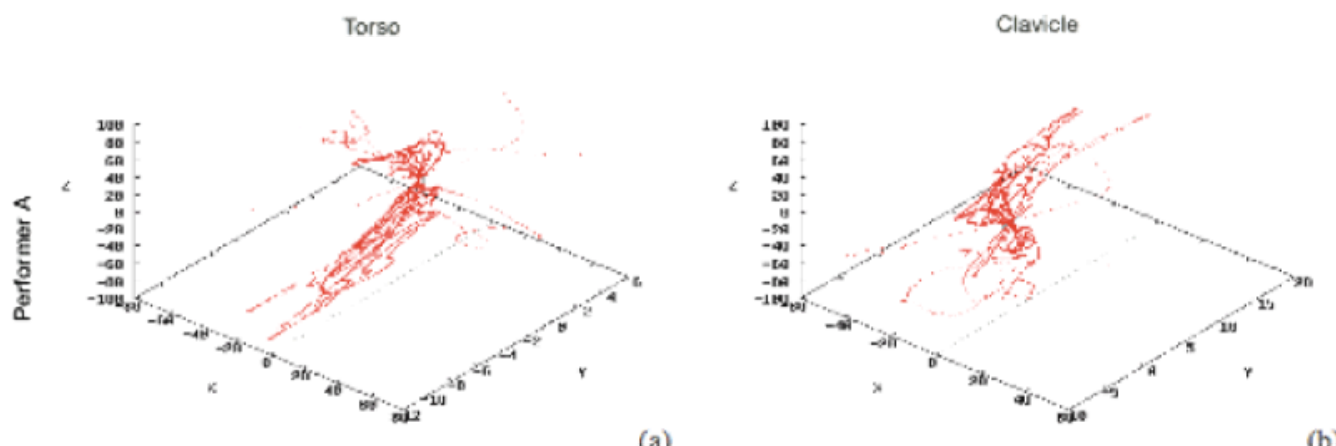

(a)
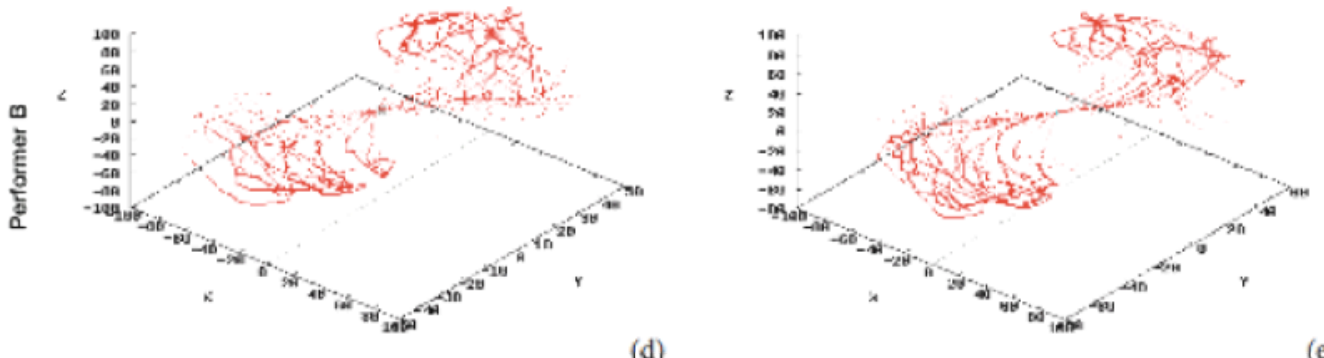

(e)

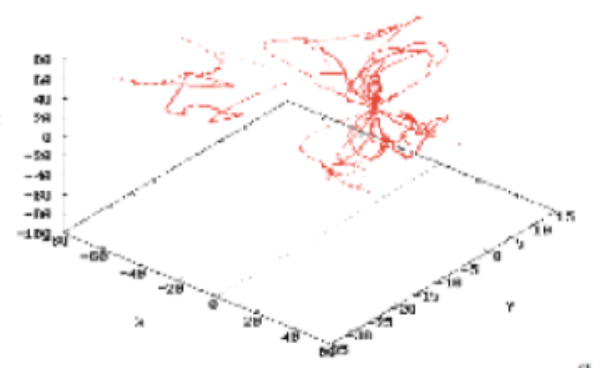

(h)
Head

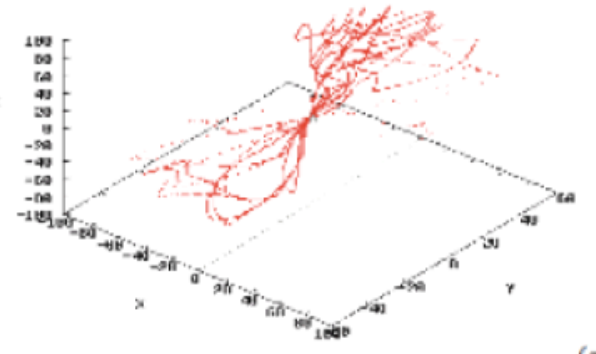

(c)

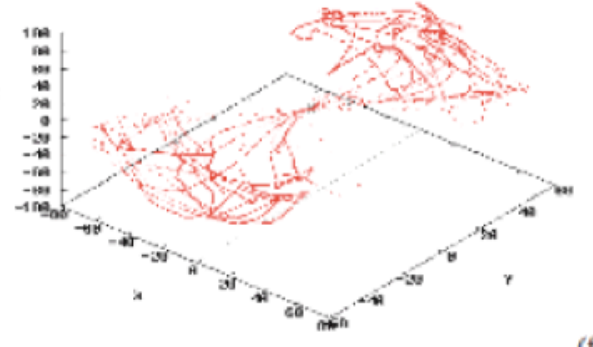

(f)

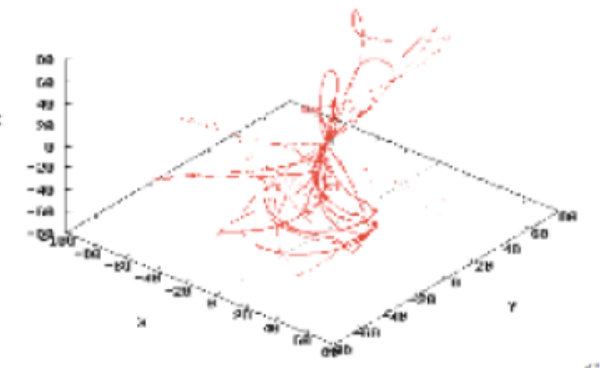

(i)

Fig. 7. Spherical analyses for the head, clavicle and torso motion of Performers A, B \& C during performances of the B minor Prelude (corresponding to motion profiles $b, c \& i$ in Fig. 5 and Appendix B). 


\section{GENERAL DISCUSSION}

Findings have shown that patterns in performance motion are directly related to pianists' individual interpretations of structure. Previous research established that pianists made repeating patterns of movement in the upper-body and head for phrases of identical (as in the A major Prelude) and extending (as in the B minor Prelude) rhythmic content (MacRitchie et al., 2013). Extending these findings, we demonstrate that variations between the shapes and form of movement patterns among performers correspond to points where individual interpretations of the music's phrasing structure differ. Furthermore, analyses for individual performers have illustrated that movement patterns vary during their performance, particularly at points of structural ambiguity. Providing novel visual representation of the performance patterns produced by head and torso motion, spherical analyses reveal the shape of the repeated phrasing motions exhibited by these nine pianists. The variability between the shapes and physical locations of these phrasing gestures refutes notions that there may exist a set gesture pattern among performers to represent phrasing. Instead findings clearly indicate that different performers generate their own highly personalised patterns of motion relating to phrasing that is repeated and altered relative to their individual interpretations of a piece's structural content. These patterns of motion, while consistently generated throughout individual performances and repeated at phrasing transitions, vary in shape, size and direction between the performances of the two pieces (Op. 28, Nos. $7 \& 6$ ). These shapes may also be seen to vary at different locations across the body, both between performers for each piece, and individually for each performer between the two pieces.

The global nature of the motion profiles, where no individual areas of the upper body or head were found to be leading performance motions (MacRitchie et al., 2013; MacRitchie, 2011), is explained by the current findings that each of the nine performers expresses repeated motion patterns simultaneously at multiple body regions throughout their performances. The nature of this expression is not identical across performers, confirming earlier proposals that performance motion is highly idiosyncratic. As a result, the patterns of motion illustrated globally through PCA and locally through spherical analyses may be interpreted as representing each individual performer's expressions of personalised phrasing gestures. Phrasing gestures may, therefore, be considered as identifiable patterns of motion that repeat relative to a performer's interpretation and intended expression of phrasing structure. While potentially differing and diverging in shape, size and direction across varying body regions, these gestures may be seen to represent a Gestalt embodiment of structure that incorporates all regions of the upper body in varying ways to corporeally describe the performer's intended expression of hierarchical levels of structure. In summary, performers use their body movements to emphasise phrasing structure and higher-order structural features that they have interpreted as being more important, rather than using the same magnitude and shape of movements consistently throughout the piece.

These global phrasing gestures are not universal in size, location or form across performers. However, they are exhibited by all performers and appear universally related to performed phrasing structure. Evidently, analyses confirm that highly skilled piano performers consistently make repeated patterns of upper-body movement relating to performed phrasing structure. These movements are highly personalised, typically taking the shape of lemniscate $(\infty)$, annodated (S), or crescent (C) shaped movements, and occasionally appearing as cruciform $(t)$, sigmate $(\Sigma)$ and ' $L$ ' shaped motions, varying in their precise dimensions both between the performers and across their performances within this data set.

By comparing the detailed analyses of nine highly skilled pianists this research has provided a quantifiable investigation into the generation of performance motion. Results indicate that global concurrent, non-sound producing movements are used by highly skilled performers to relay and emphasise their interpretations of phrasing structure and rhythmic, harmonic and melodic content. For most of the performers examined, when the new auxiliary melodic motif is developed in the B minor Prelude, patterns of phrasing motion are seen to change quite substantially from those motions representing the original $\mathrm{B}$ minor motif. This is expressed by individual performers through variations in the size and/or direction of phrasing motions or as complete changes to the shape of motions for these particular phrases. Such changes occur where the harmony alters within structurally important phrases, such as the introduction of the auxiliary motif in phrase three, the harmonic modulation into $\mathrm{C}$ major of the fifth phrase and the development of the new auxiliary motif in phrase six. Some additional influences on motion for varying interpretations of the seventh phrase are also observed. Changes in motion patterns are not solely tied to variations in pitch, as may first be assumed. Had this been the case, similar variations would have been 
expected across all nine performers corresponding to the direction and nature of pitch progression within these phrases. Instead, very different changes in patterns are observed for individual performers, with gestures often appearing as motion toward and away from the keyboard, rather than movements along the keyboard that might otherwise be interpreted as relating to pitch. Occasionally a generation of motion that is quite counter-intuitive to that required for the execution of the notes themselves is observed, such as motion patterns of the ninth performer (Performer C) who typically moves up, left and away from the keyboard as the melody climbs into the upper register of the keyboard.

Findings suggest that it may be beneficial to readdress the notion of performance motion as a corporeal reflection of moving sonic forms, as suggested by Leman (2008) and Godøy (2010). While we have seen that the patterns of motion generated by performers do adhere to the structural progression expressed audibly throughout performances, the form and variations of performance motions appear too complex simply to be representations of the pitch content of the phrase. Such movement patterns are shown here to contain rich information relating not only to the rhythm, melody and harmony of the two pieces, and how these correspond to the overall music structure, but also to each individual performer's interpretation of these features. Further to gestural representations of the structural hierarchy, an influence of individual interpretations of narrative is also implied through the observation that the shape and form of phrasing motions differ for performances of the two pieces by each individual performer. In this way, performance motions can be seen to reflect individual interpretations of how phrases mirror and answer one another, presenting diverging or novel musical ideas within the overall narrative. With no two performers generating identical patterns of phrasing motion through both global and local representations of structure, expressions of the music's structure remain highly individualised.

The findings that all performers generate repeated idiosyncratic patterns of motion relating to structural interpretations quantify and support suggestions by Desmet et al. (2012) that a performer's motions relate to audible expressions of performers' interpretations of annotations found within the score.

We extend this to consider Rink's proposals for interpretations of the B minor Prelude in particular, for example, highlighting the entry of the right hand auxiliary motif halfway through the third phrase (Rink, 2012). Features such as this may or may not be chosen by the performer to be accentuated through changing patterns of performance motion depending on their own interpretations of structure and narrative.

The patterns of motion exhibited by the performers for the B minor Prelude show marked variations in structural interpretations of the third, fifth, sixth and seventh phrases. While the current paper has touched on the potential causes for varying interpretations of phrasing structure for this piece, it has remained focused on the marked segmentations interpreted by the individual performers and on a comparison of these in relation to their expressed performance motions. Despite all performers being issued with the same edition of the Prelude, variations occur that coincide with those found within the multiple first editions of this piece (available via www.ocve.org.uk). In particular, where performers have interpreted the first bar of the sixth phrase as being separated from the remainder of the phrase, this phrasing is not always mirrored within the near identical seventh phrase, as one might expect. In the French and English first editions of this Prelude, the first bar of the sixth phrase has a separate slur whereas that of the seventh is joined to what follows, rather than these phrase markings conforming to one another as in the German first edition. These same variations in structural interpretation are evident in some performers' notations gathered during interview, corresponding directly to variations observed within their performance motions.

It would be possible to apply an adaptation of the SoP model, proposed by Rasamimanana (2012), to consider non-technical performance motion and the range of performance gestures identified here. Using spherical motion trajectories as the input data for parsing performance gesture, rather than acceleration data, may help to develop clearer and more accurate identification, quantification and categorisation of performance gestures. By taking directionality and the changing magnitude of the performance motion vector into account, it may be possible to effectively identify and model performance gestures relative to expressions of structural features and segmentations.

Motion patterns that previously may have been attributed to noise within the motion signal, or to other factors influencing individualised performance (such as mood, individual performance styles, etc), have been clearly identified here as relating to stylistic and personalised interpretations of musical structure by performers. Therefore, future research considering the causal and functional roles of performance motion could benefit substantially from identifying the structural interpretations and intended expressions made by performers for the repertoire under consideration. The particular shaping and form of performers' phrasing gestures examined within the current paper support Caramiaux et al.'s (2012) findings that 
performers made circling motions with their clarinet bell relative to timing structures within their performances. In addition, we demonstrate here that gestural patterns vary in their overall shape, size and direction across performers and performances, and in their hierarchical importance, but are all formed by the generation of repeating curved motions expressed throughout the body and rotating around a performer's polar axis. The global representation of phrasing structure throughout each performer's upper body motion, combined with the evidence that while the shape and trajectory of performance gestures may vary across body regions, their temporal relations to phrasing structure and the accentuation of higher-order features do not, supports findings that performers exhibit a unique mapping of expression relative to timing (Palmer, 1989). We see the results of these varying interpretations as differences in the way performers move their upper bodies, with movement dispersed throughout the extended regions of the body from the central point of motion, relating to Davidson's reference to an expressive 'centre of moment' in music performance (2007).

This study has implications for the assessment, analysis and categorisation of non-technical performance gestures within performance science, music pedagogy and gestural research more generally. The use of spherical coordinates to visualise patterns of repetitive motions and their relation to structure could be applied within future studies to examine motion trajectories across a range of instruments, performers and performances, as well as to a variety of compositions, to further explore the relationships between performance style, genre and the shapes of gestures generated by performers. Future research could compare the gestural shapes created by different instrumentalists to explore the relation of nontechnical phrasing gestures to technical motion and the impact of instrumental expertise on performance movements. The demonstration that structural interpretation shapes non-technical performance movement may be extended to consider larger pieces from varying genres. This would confirm the relationship between non-technical performance movement and the specific melodic, harmonic, and rhythmic content of each phrase. This paper has confirmed that performers use idiosyncratic gestures to express phrasing structure. The application of the methods used here may inform current gesture segmentation and parsing models to extend investigations into the underlying cognitive and behavioural functions of human body movement in both musical and social contexts.

\section{CONCLUSION}

All performers generate idiosyncratic movements that relate to individual interpretations of phrasing structure. These repeated movements typically, but not necessarily always, take the shape of lemniscate, annodated or crescent movements traced through head, clavicle and torso motions. Shapes of phrasing gestures vary between performers and between performances; the shape and direction of these change specifically with regard to structural interpretation and phrase hierarchy. Results build upon previous research (MacRitchie et al. 2013) demonstrating that there does not exist a particular movement shape that may be termed as embodying phrasing, but rather that individual pianists universally embody phrasing structure in their own idiosyncratic manner relating to personal interpretations of structure.

This study has drawn upon the performers' non-sound-producing, concurrent movements to examine the extents and limits to which inferences can be drawn regarding musical structure. In many cases, such as the B minor Prelude presented here, the musical structure is (deliberately or otherwise) not made explicit through score analysis, leading to diverse, and sometimes contradictory, expert opinions. Analysis of performers' gestures uncovers knowledge implicit in expert practitioners' performance study, and is shown to be repeatably and reliably useful in determining intent. After a sufficient corpus of gesture recordings has been made, the information generated will be helpful in tracking changes in musical interpretation in contemporary performance practice.

\section{NOTES}

[1] B. Buck and J. MacRitchie hold joint first authorship for this paper. Correspondence may be directed to B.Buck.1@research.gla.ac.uk and jennifer.macritchie@conservatorio.ch.

[2] Performers have been shown to alter their corporeal movements to effect the timbre or tone of the notes produced through variations in bowing movements or positioning of the bell of a clarinet, for example 
(Young, 2007; Wanderley et al., 2005). Such movements have been categorised as being sound-facilitating or -modulating (Jensenius et al. 2010) sound-accompanying (Cadoz \& Wanderley, 2000; Godoy, 2010) or ancillary (Wanderley \& Depalle, 2005; Caramiaux et al., 2012; Desmet et al., 2012)

[3] Becking claimed that the distribution of these weights varied from composer to composer, reflecting different composers' "management of gravity", which in turn Becking suggested represented their attitudes to physical reality. Becking aimed to discern a connection between the composer's philosophical and conceptual ideas, represented through their music, and the patterns of motion that they evoked. While Becking's methods were entirely subjective and arguably circular, his examples of movement curves seem to reflect the patterns of motion that are arising within motion research today. Discussions of his work can be found in Repp (1993), Sievers (1924) and Becking's own thesis, published 1928.

[4] Use of this high-action keyboard was necessary due to limitations when using the stationary Vicon system without the ready availability of an acoustic piano nearby.

[5] Courtesy of Prof. F. Pollick of The University of Glasgow's School of Psychology.

[6] Reproduced with permission from Musicae Scientiae.

[7] Actual phrasing boundaries were located relative to the termination of the last note of each phrase as it appeared within the recorded audio stream of each individual performance. Motion data was then normalised in time and mapped to represent changes in motion relative to these boundaries on a timescale of 0 to 1 , with each decile in time representing a complete phrase segmentation in the audio dimension.

[8] When indicating phrasing segmentations within such graphical representations the segment referents often appear as clusters, demonstrating that the shape and form of phrasing motions overlay, following similar shapes and trajectories within an individual performance.

[9] Performer 4 makes small swaying motions first toward and then along the keyboard during the majority of phrases, sweeping along the full length of the keyboard twice during her performance of the A major Prelude.

\section{ACKNOWLEDGEMENTS}

The authors would like to thank Prof. Frank Pollick at the University of Glasgow for access to his Vicon motion capture setup, Lukasz Piwek for his time and help in the setup and recordings of the pianists, and Charlotte Woolley for her assistance and support. This research was partially funded through EPSRC doctoral training grants of the primary authors.

\section{REFERENCES}

Broughton, M., \& Stevens, K. (2009). Music, movement and marimba: An investigation of the role of movement and gesture in communicating musical expression to an audience. Psychology of Music, Vol. 37, No. 2, pp. 137-153.

Buck. B. (2013) Gestural communication of music structure through solo piano performance. Forthcoming Doctoral thesis. University of Glasgow, UK.

Cadoz, C., \& Wanderley, M.M. (2000). Gesture-Music. In: M.M. Wanderley \& M. Battier (Eds.), Trends in Gestural Control of Music. Paris: Ircam Centre Pompidou, pp. 71-93.

Camurri, A., Mazzarino, B., Ricchetti, M., Timmers, R., \& Volpe, G. (2004). Multimodal analysis of expressive gesture in music and dance performers. In: A. Camurri \& G. Volpe (Eds.), Gesture-based Communication in Human-Computer Interaction. Berlin/Heidelberg: Springer-Verlag, pp. 20-39. 
Caramiaux, B., Wanderley, M.M., \& Bevilacqua, F. (2012). Segmenting and parsing instrumentalists' gestures. Journal of New Music Research, Vol. 41, No.1, pp. 13-29.

Clayton, M. (2007). Time, gesture and attention in khayl performance. Asian Music, Vol. 38, No. 2, pp. 7196.

Clarke, E.F., \& Davidson, J.W. (1998). The body in performance. In: W. Thomas (Ed.), CompositionPerformance-Reception. Aldershot: Ashgate, pp. 74-92.

Cutti, A., Paolini, G., Troncossi, M., Cappello, A., \& Davalli, A. (2005). Soft tissue artefact assessment in humeral axial rotation. Gait and Posture, Vol. 21, pp. 341-349.

Cutting, J.E., Proffitt, D.R., \& Kozlowski, L.T. (1978). A biomechanical invariant for gait perception. Journal of Experimental Psychology: Human Perception and Performance, Vol. 4, pp. 357-72.

Dahl, S., \& Friberg, A. (2007). Visual perception of expressiveness in musicians' body movements. Music Perception, Vol. 24, No.5, pp. 433-454.

Dahl, S., Bevilacqua, F., Bresin, R., Clayton, M., Leante, L., Poggi, I., \& Rasamimanana, N. (2010). Gestures in Performance. In: R.I. Godøy \& M. Leman (Eds.), Musical Gestures: Sound, Movement, and Meaning. New York: Routledge, pp. 36-68.

Davidson, J.W. (1993). Visual perception of performance manner in the movements of solo musicians. Psychology of Music, Vol. 21, No. 2, pp. 103-113.

Davidson, J.W. (1994). What type of information is contained in the body movements of solo musician performers? Journal of Human Movement Studies, Vol. 6, pp. 279-301.

Davidson, J.W. (2007). Qualitative insights into the use of expressive body movement in solo piano performance: a case study approach. Psychology of Music, Vol. 35, No.3, pp. 381-401.

Desmet, F., Nijs, L., Demey, M., Lesaffre, M., Martens, J-P., \& Leman, M. (2012). Assessing a clarinet player's performer gestures in relation to locally intended musical targets. Journal of New Music Research, Vol. 41, No.1, pp. 31-48.

Dodson, A. (2011). Expressive timing in expanded phrases: an empirical study of recordings of three Chopin Preludes. Music Performance Research, Vol. 4, pp. 2-29.

Godøy, R.I., \& Jensenius, A. (2009). Body movement in music information retrieval. In: K. Hirata \& G. Tzanetakis (Eds.), 10th International Society for Music Information Retrieval Conference. Kobe, Japan: ISMIR, pp. 45-50.

Godøy, R.I. (2010). Gestural affordances of musical sound. In: R.I. Godøy \& M. Leman (Eds.). Musical Gestures: Sound Movement, and Meaning. New York: Routledge, pp. 103-125.

Goebl, W. \& Palmer, C. (2009). Synchronization of timing and motion among performing musicians. Music Perception, Vol. 26, No.5, pp. 427-438.

Goldin-Meadow, S. (1993). Transitions in concept acquisition: Sing the hand to read the mind. Psychological Review, Vol. 100, No. 2, pp. 279-297.

Jensenius, A., Wanderley, M., Godøy, R.I, \& Leman, M. (2010). Musical gestures: Concepts and methods in research. In: R.I. Godøy \& M. Leman (Eds.), Musical Gestures: Sound, Movement, and Meaning. New York: Routledge, pp. 13-35.

Keller, P.E., \& Appel, M. (2010). Individual differences, auditory imagery, and the coordination of body movements and sounds in musical ensembles. Music Perception, Vol. 28, No.1, pp. 27-46. 
Kendon, A. (2004). Gesture. Visible Action as Utterance. Cambridge: Cambridge University Press.

King. E., \& Ginsborg, J. (2011). Gestures and glances: Interactions in ensemble practice. In: A. Gritten \& E. King (Eds.), New Perspectives on Music and Gesture. Farnham: Ashgate, pp. 179-202.

Leman, M. (2008). Embodied Music Cognition and Mediation Technology. Cambridge, MA: MIT Press.

MacRitchie, J. (2011). Elucidating musical structure through empirical measurement of performance parameters. Doctoral thesis, University of Glasgow.

MacRitchie, J., Buck, B., \& Bailey, N.J. (2013) Inferring musical structure through bodily gestures. Musicae Scientiae, Vol. 17, No.1, pp. 86-108.

McNeill, D. (2005). Gesture and Thought. Chicago, Illinois: University of Chicago Press.

Munoz, E.E. (2007). When gesture sounds: Bodily significance in musical performance. In: A. Williamon \& D. Coimbra (Eds.), International Symposium on Performance Science 2007. European Association of Conservatoires (AEC), Utrecht, The Netherlands, pp. 55-60.

Naveda, L., \& Leman, M. (2008). Representation of Samba dance gestures, using a multi-modal analysis approach. In: 5th International Conference on Enactive Interfaces (ENACTIVE'08). Pisa, Italy, pp. 68-74.

Nusseck, M., \& Wanderley, M.M. (2009). Music and motion - how music-related ancillary body movements contribute to the experience of music. Music Perception, Vol. 26. No. 4, pp. 335-353.

Palmer, C. (1989). Mapping musical thought to musical performance. Journal of Experimental Psychology: Human Perception and Performance, Vol. 15, No. 12, pp. 331-346.

Palmer, C. (1997). Music performance. An Annual Review of Psychology, Vol. 48, pp. 115-138.

Platz, F., \& Kopiez, R. (2012). When the eye listens: A meta-analysis of how audio-visual presentation enhances the appreciation of music performance. Music Perception, Vol. 30, No.1, pp. 71-83.

Rasamimanana, N. (2012). Towards a conceptual framework for exploring and modelling expressive musical gestures. Journal of New Music Research, Vol. 41, No.1, pp. 3-12.

Repp, B. (1993). Musical Motion: Some historical and contemporary perspectives. In: A. Friberg, J. Iwarsson, E. Jansson, \& J. Sundberg (Eds.), Stockholm Music Acoustics Conference. Stockholm, pp. 128135.

Rink J. (2012). Personal communication. CMPCP's Music and Shape Conference, University of London, July 12-14, 2012.

Rink, J. (2002). Analysis and (or?) performance. In: J. Rink (Ed.), Musical Performance: A Guide to Understanding. Cambridge: Cambridge University Press, pp. 35-58.

Sievers, E. (1924). Ziele und Wege der Schal/analyse. Heidelberg: Carl Winter's Universitätsbuchhandlung. Cited in: Repp, B. (1993). Musical Motion: Some historical and contemporary perspectives. In: A. Friberg, J. Iwarsson, E. Jansson, \& J. Sundberg (Eds.), Stockholm Music Acoustics Conference. Stockholm, pp. 128135.

Styns, F., \& Van Noorden, L. (2006). Some basic observations on how people move on music and how they relate to music movement. In: A. Gritten \& E. King (Eds.), Second International Conference on Music and Gesture. Manchester: Northern College of Music.

Thompson, M., \& Luck, G. (2008). Exploring relationships between expressive and structural elements of music and pianist's gestures. In: E. Cambouropoulos, R. Parncutt, M. Solomos, D. Stefanou, \& C. Tsougras (Eds.), Fourth Conference on Interdisciplinary Musicology (CIM08). Thessaloniki, Greece. 
Thompson, M., \& Luck, G. (2011). Exploring relations between pianists' body movements, their expressive intentions, and structural elements of the music. Musicae Scientiae, Vol.16, No.1, pp. 19-40.

Van Noorden, L. (2010). The functional role and bio-kinetics of basic and expressive gestures in activation and sonification. In: R.I. Godøy \& M. Leman (Eds.), Musical Gestures: Sound, Movement, and Meaning. New York: Routledge, pp. 36-68.

Vines, B.W., Krumhansl, C.L., Wanderley, M.M., \& Levitin, D.J. (2006). Cross-modal interactions in the perception of musical performance. Cognition, Vol. 101, pp. 80-113.

Vines, B.W., Wanderley, M.M., Nuzzo, R.L., Levitin, D.J., \& Krumhansl, C.L. (2004). Performance gestures of musicians: What structural and emotional information do they convey? In: A. Camurri, \& G. Volpe (Eds.), Gesture-Based Communication in Human-Computer Interaction. Berlin/Heidelberg: Springer-Verlag, pp. 468-478.

Wanderley, M.M. (2002). Quantitative analysis of non-obvious performer gestures. In: I. Wachsmuth \& T. Sowa (Eds.), Gesture and Sign Language in Human-Computer Interaction. Berlin/Heidelberg: SpringerVerlag, pp. 241-253.

Wanderley, M.M. \& Depalle, P. (2005). Gestural control of sound synthesis. In: G. Johannsen (Ed.), IEEE. Special Issue on Engineering and Music - Supervisory Control and Auditory Communication, Vol. 9, No. 4, pp. 632-644.

Wanderley, M.M., Vines, B., Middleton, N., McKay, C., \& Hatch, W. (2005). The musical significance of clarinettists' ancillary gestures: An exploration of the field. Journal of New Music Research, Vol. 34, pp. 97-113.

Young, D. (2008). Classification of common violin bowing techniques using gesture data from a playable measurement system. In: A. Camurri, S. Serafin, \& G. Volpe (Eds.), International Conference on New Interfaces for Musical Expression (NIME). Genoa, Italy, pp. 44-48. 


\section{APPENDIX}

Appendix A: Scores - first phrase of A major Prelude and full score for B minor Prelude (with typical analysis of phrasing marked for phrase reference within the text)

Figure a.: Chopin's Prelude in A major (Op.28:7); opening phrase.

The initial, opening phrase depicted here demonstrates the strict 2-bar rhythmic identity of all eight phrases of the A major Prelude.

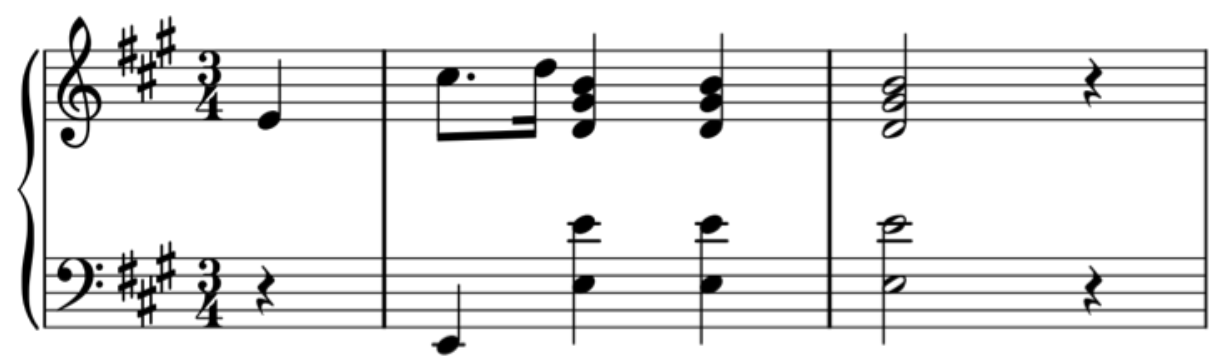

Figure $b$ (next page).: Chopin's Prelude in B minor (Op.28:6); full score.

The scores of Chopin's Prelude in B minor is presented overleaf. Horizontal and vertical lines outwith the musical score indicate the eight phrasing boundaries as described by the musical analysis in body of the text. Score images have been obtained from http://mutopiaproject.org, where unrestricted modification is permitted as editions have been contributed to the public domain. 


\section{Prelude}

'Tolling Bells'
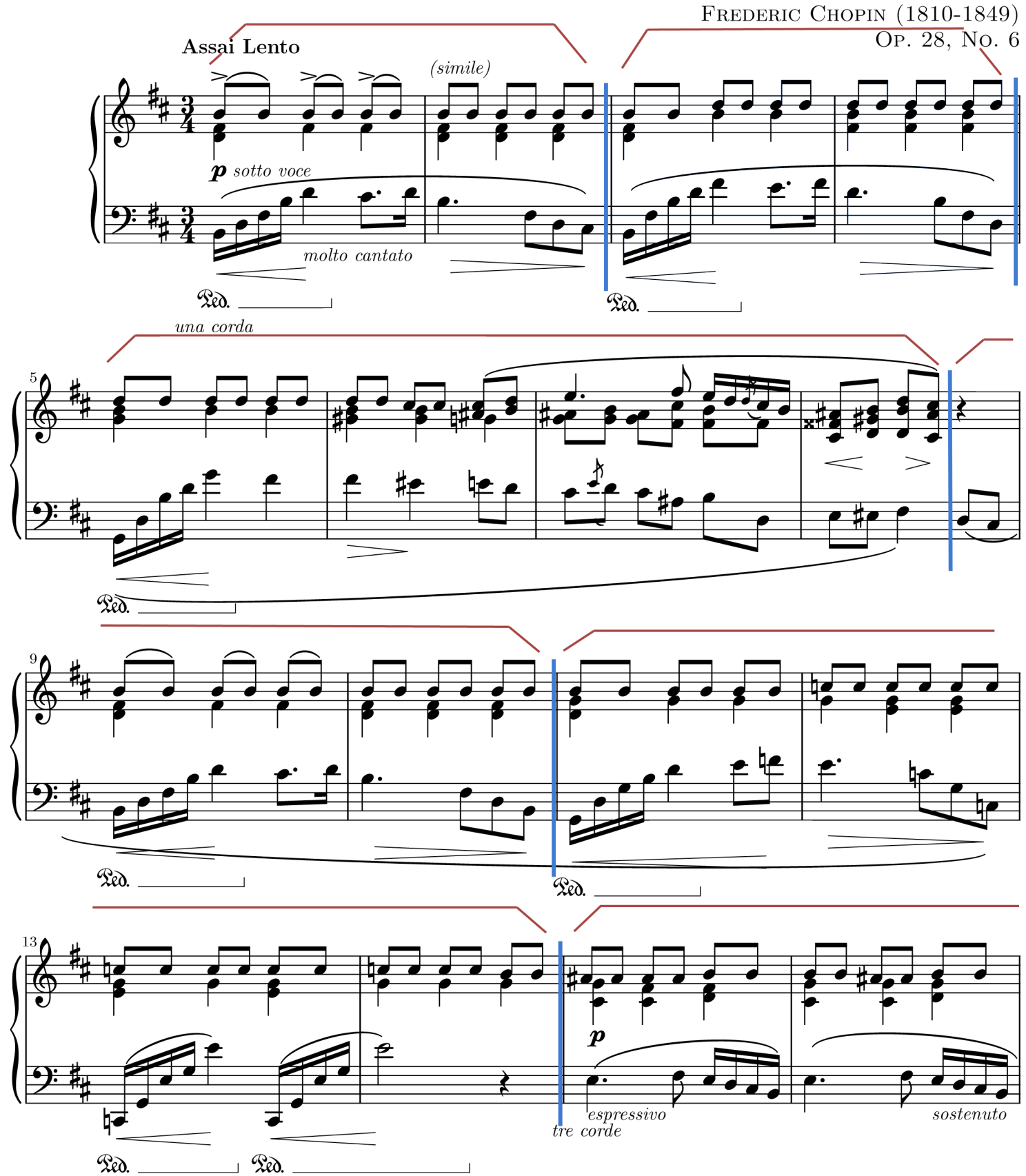


Appendix B: PCA Motion Profiles for A major Prelude* - individual motion profiles showing combinations of principal components explaining $\geq 90 \%$ of performance movements exhibited by each of the nine performers (Performers 1-9 represented as plots a-i in figure)

*(reproduced with permission from Musicae Scientiae)
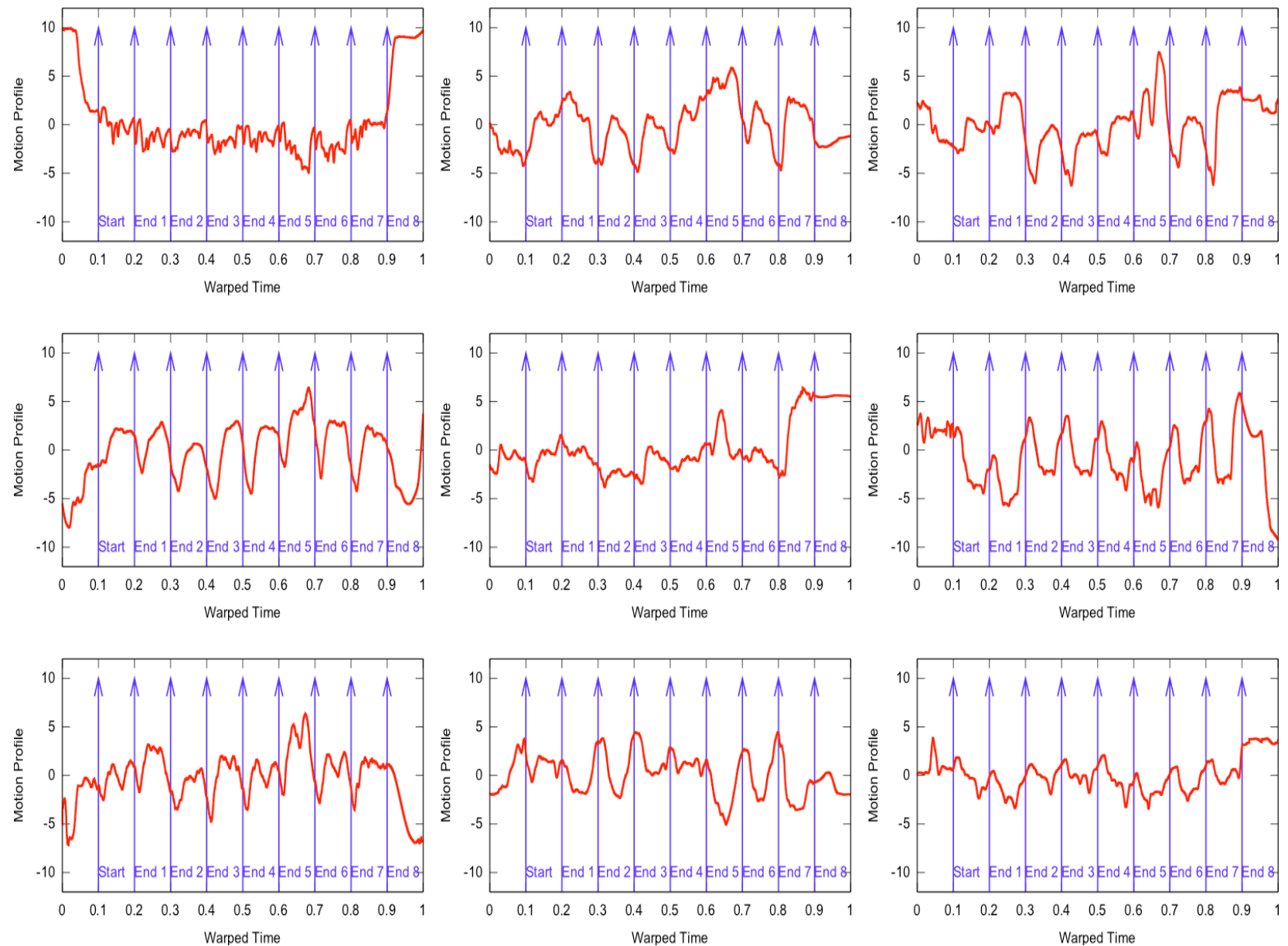